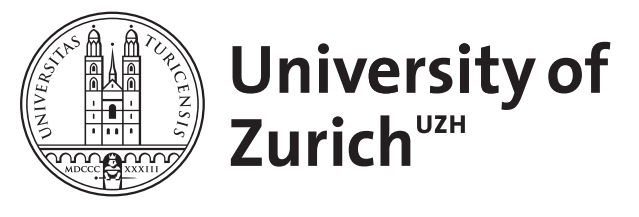

\title{
Reversibility in dynamic coordination problems
}

\author{
Kováč, Eugen ; Steiner, Jakub
}

\begin{abstract}
We study a dynamic coordination process in which agents are uncertain about the actions of their fellow agents, and anticipate strategically relevant information. Because of the uncertainty and learning, (ir)reversibility of actions has important strategic consequences. We find that the reversibility option can either enhance or hamper efficient coordination, and we characterize the direction of the effect based solely on simple features of the coordination problem. The analysis is based on the following generalization of the Laplacian property known from static global games: agents at the beginning of the dynamic game act as if they were entirely uninformed about the aggregate play of fellow agents in each stage of the coordination process.
\end{abstract}

DOI: https://doi.org/10.1016/j.geb.2012.10.014

Posted at the Zurich Open Repository and Archive, University of Zurich ZORA URL: https://doi.org/10.5167/uzh-174739

Journal Article

Accepted Version

Originally published at:

Kováč, Eugen; Steiner, Jakub (2013). Reversibility in dynamic coordination problems. Games and Economic Behavior, 77(1):298-320.

DOI: https://doi.org/10.1016/j.geb.2012.10.014 


\title{
Reversibility in Dynamic Coordination Problems*
}

\author{
Eugen KovÁČ \\ JAKUB STEINER ${ }^{\ddagger}$ \\ University of Bonn \\ Northwestern University
}

May 11, 2012

\begin{abstract}
We study a dynamic coordination process in which agents are uncertain about the actions of their fellow agents, and anticipate strategically relevant information. Because of the uncertainty and learning, (ir)reversibility of actions has important strategic consequences. We find that the reversibility option can either enhance or hamper efficient coordination, and we characterize the direction of the effect based solely on simple features of the coordination problem. The analysis is based on the following generalization of the Laplacian property known from static global games: agents at the beginning of the dynamic game act as if they were entirely uninformed about the aggregate play of fellow agents in each stage of the coordination process.

JEL classification: $\mathrm{C} 7, \mathrm{D} 8$.

Keywords: Delay, Exit, Global Games, Laplacian Belief, Learning, Option, Reversibility.
\end{abstract}

${ }^{*}$ We thank the editor and the anonymous referees, George-Marios Angeletos, Leonardo Felli, Paul Heidhues, Tai-Wei Hu, Sergei Izmalkov, Daniel Krähmer, Nicolas Melissas, Tymofiy Mylovanov, John Moore, Marcin Pȩski, Carolyn Pitchik, Muhamet Yildiz, seminar participants at CERGE-EI, LSE, MIT, UCL, University of Berlin, Bern, Bielefeld, Bonn, Bratislava, Edinburgh, Mannheim, Kiev, Northwestern and Toronto as well as participants of the conferences EEA-ESEM 2008, ES NASM 2009, ESWC 2010, Games 2008, SED Annual Meeting 2008, Verein für Socialpolitik 2011, and the workshops at ESSET 2008, ESI Jena 2009, and GRIPS 2008 for helpful comments. We especially appreciate detailed comments from Kohei Kawamura, József Sákovics, and Colin Stewart.

${ }^{\dagger}$ Department of Economics, University of Bonn, Adenauerallee 24-26, 53113 Bonn, Germany; e-mail: eugen.kovac@uni-bonn. de; URL: www. uni-bonn.de/ kovac.

${ }^{\ddagger}$ Kellogg School of Management, MEDS, Northwestern University, 2001 Sheridan Road, Evanston, IL 60208; j-steiner@kellogg.northwestern.edu 


\section{Introduction}

An agent at the outset of an economic crisis faces strategic uncertainty. She does not know the future of the economy because she is uncertain how fellow agents perceive the odds of the crisis and hence how they will react. As the crisis unfolds, the agent receives strategically valuable information upon which she may reconsider her earlier decisions. Therefore, her early investment decisions and, at the macroeconomic level, the aggregate actions and the final outcome of the crisis may crucially depend on the reversibility of the early actions. The effects of reversibility are complex. On the one hand, reversibility allows agents to react early to crises, effectively alleviating fears of entry. On the other hand, reversibility may lead to panic exit waves that deepen the late stages of crises. The expectation of the panic may exacerbate anticipatory fears.

Our starting observation, that of strategic uncertainty at the outset of crisis, is well formalized in the global games literature. A global game is a static, incomplete information coordination game. Agents receive private signals about an underlying economic fundamental and, in the unique equilibrium, those with signals above a certain threshold invest. Our uncertain agent fearing the crisis corresponds to the threshold type who, being on the boundary between the investing and non-investing types, is uncertain about the aggregate investment. A key to the solution of static global games is the so-called Laplacian Property which claims that the belief held by the threshold type is very simple: she believes that the aggregate investment is distributed uniformly across all feasible investment levels. To emphasize the connection to Laplace's principle of insufficient reason, Morris and Shin (2003) dub such a belief Laplacian. In this paper we generalize this Laplacian property for dynamic environments.

Because of their tractability, static global games are often applied even at the cost of suppressing dynamic features of the analyzed problem. For example, Morris and Shin (2004) study debt crises as coordination failures arising among creditors. Treating the early investments of the creditors as exogenous, they allow them to prematurely withdraw their funds in the interim stage of the project. The modelled interaction thus becomes a simultaneous-move game in which investors prefer to withdraw if they believe that too many fellow investors are withdrawing. The authors apply the static global game framework and find investors' panic in the unique equilibrium - a wave of inefficient interim exits. It is thus natural to ask whether the exit option should be provided, and at what cost. Such a question requires a dynamic model with endogenous entry decisions because policies affecting the exit option - the liquidity of investment - will also affect the entry. This paper provides such framework.

We study a dynamic coordination game in which agents decide whether to invest in a project consisting of two stages. At the beginning of the first stage, all agents make a binary investment decision based on their initial private information. During the first 
stage, agents learn additional private information and they can reverse the initial decision in between the two stages. More precisely, one of the two available actions, investment (risky action) or no investment (safe action), is irreversible and the other is reversible. This induces an option value of the reversible action. We call the reversibility of the risky investment the exit option and reversibility of the safe action the delay option.

We study the effects of the reversibility option by comparing the equilibria of the dynamic game with that of a benchmark static game without the option. Such a comparison is not straightforward because it has to deal with strategic effects. In non-strategic decision problems, the reversibility option is unambiguously beneficial, as it allows for adjustment to the arriving information. However, in strategic environments, reversibility may lead to fear-driven panic exits or to investment delays. Thus, reversibility may either hamper or enhance coordination on investment.

The value of the option to reverse early action is an endogenous, equilibrium object in our model. Our main technical insight is that it can be partially characterized by the use of the Laplacian property generalized to dynamic games. We find that the threshold type at the beginning of the game forms an expectation of the option value as if she had no information about the investment level in the late stage of the project.

Thanks to the generalized Laplacian property, the characterizing of the reversibility effects becomes simple. As the property holds in both the static and dynamic games, we need not worry about the differences in the equilibrium beliefs across the two games and we can evaluate the differences in equilibrium actions based solely on certain simple mechanistic properties of the investment project.

We find that the strategic consequences of the option depend on the intertemporal payoff structure. Agents receive profit for each project stage in which they participate and maximize the total sum of profits. We say that payoffs exhibit forward spillovers if production involves inertia, such that the profit in the late stage depends not only on the late but also on the early investment level. Payoffs exhibit backward spillovers if the profit in the early stage depends not only on the early but also on the late investment level. ${ }^{1}$ Using this terminology, the effects are as in Table 1: the exit option enhances efficient coordination in projects with no backward spillovers and hampers efficient coordination in projects with no forward spillovers. The delay option has the opposite effects. As a corollary, neither the exit nor the delay option has any effect in projects without both backward and forward spillovers.

We share a focus on the effects of reversibility options on investment decisions with McDonald and Siegel (1986) or Dixit and Pindyck (1994), but we differ in the source of uncertainty. Their work on single-person investment decisions with delay option considers

\footnotetext{
${ }^{1}$ Backward spillovers arise, for instance, if agents cannot fully exit the project or under schemes which redistribute profits among the investors.
} 


\begin{tabular}{cc|cc}
\hline \multicolumn{2}{c}{ payoff spillovers } & \multicolumn{2}{c}{ option to } \\
backward & forward & exit & delay \\
\hline$\checkmark$ & $\times$ & more failures & fewer failures \\
$\times$ & $\checkmark$ & fewer failures & more failures \\
$\times$ & $\times$ & \multicolumn{2}{|c}{ irrelevance result } \\
\hline
\end{tabular}

Table 1: Effect of the reversibility option on the occurrence of coordination failures.

uncertainty coming from exogenous shocks. In our model, uncertainty is endogenous and strategic as the agents are uncertain about others' actions.

Our paper belongs to a booming literature on dynamic global games. One strand of this literature emphasizes the intertemporal tradeoffs of agents facing frictions in an evolving environment (Burdzy, Frankel and Pauzner 2001, Levin 2001, or Chassang 2010). A second strand studies public learning in a stable environment and emphasizes equilibrium multiplicity induced by observation of an endogenously chosen public policy (Angeletos, Hellwig, and Pavan 2006), observation of prices (Angeletos and Werning 2006), or observation of earlier coordination outcomes (Angeletos, Hellwig, and Pavan 2007).

Our paper also belongs to yet another stream of dynamic global games literature in which the environment is fixed, but the reversibility option has a value induced by learning (Heidhues and Melissas 2006, Dasgupta 2007, and Dasgupta, Steiner, and Stewart 2012). The learning is private, and hence equilibrium uniqueness is preserved, which facilitates the characterization of the reversibility effects. The generalized Laplacian property described in this paper unifies this characterization across a large class of settings without resorting to specific payoff functions and error distributions assumed in the above papers.

One of the dynamic effects studied in the literature but not addressed here is that investment by one agent can trigger investment of her fellow agents either through signaling or via complementarities (see Corsetti, et al. 2004 or Hörner 2004 within the global games literature, and Chamley and Gale 1994, Gale 1995, or Gul and Lundholm 1995 outside of the global games literature). Our model abstracts from informational externalities as we assume that the amount of information revealed during the coordination process is independent of agents' actions. Moreover, our agents are small and therefore cannot individually trigger the investments of others.

Our model provides an added value in situations in which strategic uncertainty plays a central role. For a better illustration, let us compare our approach to that of Gale (1995). He considers a small population of agents with irreversible investment and a delay option. Under perfect observability of actions, agents can coordinate efficiently, as each agent is eager to invest early in order to induce the investments of others. In large populations, when individual actions are not observable and agents are uncertain of aggregate actions, our model predicting inefficient coordination may be a better fit.

Questions on the timing of the exit similar to ours have also been studied outside of 
the global games literature. Abreu and Brunnermeier (2003) devise a model that explains the persistence of asset bubbles and Brunnermeier and Morgan (2010) analyze delay in clock games (a class of games where an agent's payoff depends on the time of exit). The methodology used in both these papers, however, differs from ours in that their models do not exhibit strategic complementarities.

The organization of the paper is as follows. Section 2 introduces the model; Section 3 contains the main technical contribution of the paper - it describes the generalized Laplacian property in dynamic games. The Laplacian property holds in a monotone, symmetric strategy profile, and indeed in Section 4 we prove that the game exhibit unique monotone, symmetric equilibrium, making thus the Laplacian property applicable. Section 5 identifies the strategic effects of the reversibility option by comparing equilibria across the dynamic game and the static benchmark. Section 6 contains a brief extension to settings with multiple rounds. In Section 7 we discuss our modeling assumptions. Most of the proofs are relegated to the Appendix.

\section{Model}

We study a dynamic, binary-action game with one of the two actions being reversible and the other irreversible. For the sake of concreteness we focus on the game with reversible investment - on the game with exit option. The analysis of the game with reversible safe action - the game with delay option — is symmetric, and is discussed in Section 7.
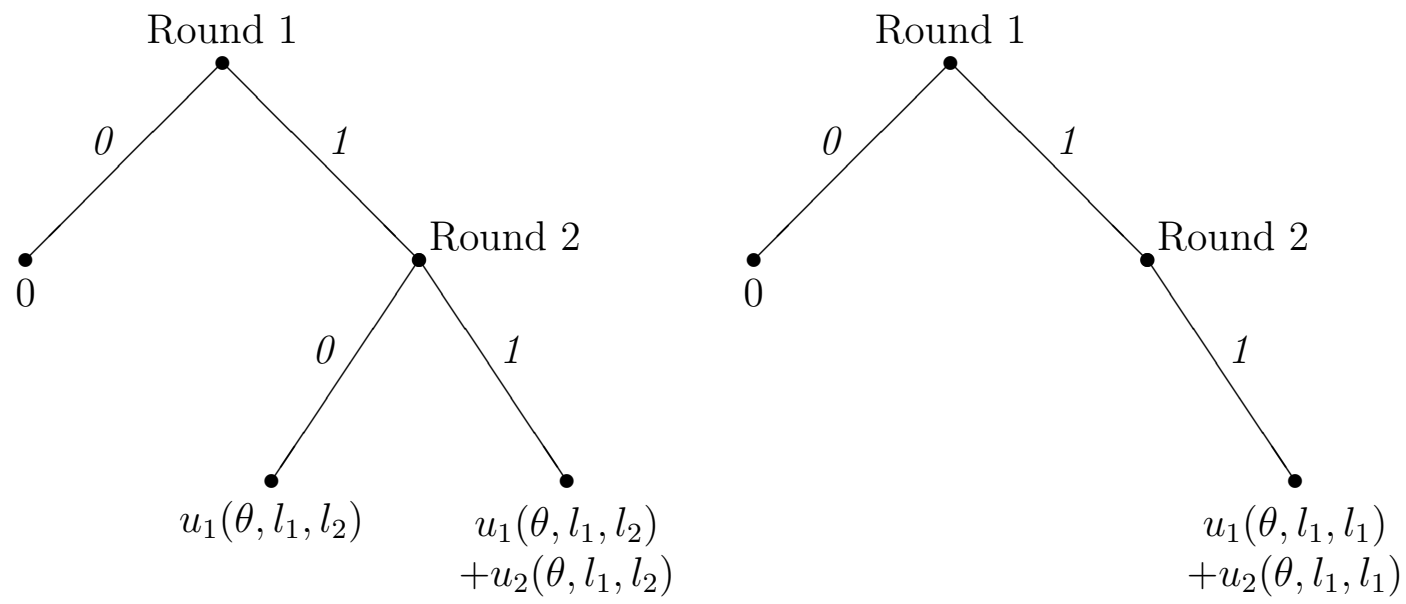

Figure 1: Decision tree in the dynamic game $\Gamma_{d y n}$ (left) and in the benchmark static game $\Gamma_{s t}$ (right). Moves of Nature and of fellow agents are not depicted.

There is a common investment project with two production stages and continuum of agents indexed by $i \in[0,1]$. Each production stage is preceded by agents' investment decisions. Before the first stage, in round 1, all agents simultaneously choose between entering the project, $a_{1}^{i}=1$ (also called investing, or risky action), or not investing, 
$a_{1}^{i}=0$ (safe action). Agents who chose the safe action, $a_{1}^{i}=0$, reach their final node and receive a payoff 0 . Entrants make a new decision in between the two production stages - in round 2. They choose simultaneously whether to exit or stay in the project, $a_{2}^{i} \in\{0,1\}$. Let $l_{1}$ denote the measure of agents choosing $a_{1}^{i}=1$ in round 1 , and $l_{2}$ the measure of agents choosing action 1 in both rounds, $a_{1}^{i}=a_{2}^{i}=1$.

The payoffs depend on three components: economic fundamental $\theta$, aggregate investment in the first round $l_{1}$, and aggregate investment in the second round $l_{2}$. The payoff for investing and exiting, $\left(a_{1}, a_{2}\right)=(1,0)$, is $u_{1}\left(\theta, l_{1}, l_{2}\right)$. The payoff for investing and staying, $\left(a_{1}, a_{2}\right)=(1,1)$, is $u_{1}\left(\theta, l_{1}, l_{2}\right)+u_{2}\left(\theta, l_{1}, l_{2}\right)$. We interpret $u_{1}$ and $u_{2}$ as payoffs for participation in stage 1 and 2 . They are real-valued functions, defined on the domain $\left\{\left(\theta, l_{1}, l_{2}\right) \in \mathbb{R} \times[0,1] \times[0,1]: l_{2} \leq l_{1}\right\}$. Both payoff functions are continuous in all arguments. $^{2}$ The additive payoff structure is without loss of generality. To avoid ambiguity in exposition, we assume that indifferent types choose action 1.

We now impose payoff restrictions common in the global games literature under which both the static and the dynamic game exhibit unique symmetric, monotone equilibrium. In particular, we assume that for $t=1,2$ the following assumptions are satisfied.

A1 Strict State Monotonicity: $u_{t}\left(\theta, l_{1}, l_{2}\right)$ is increasing in $\theta$.

A2 Weak Action Monotonicity: $u_{t}\left(\theta, l_{1}, l_{2}\right)$ is non-decreasing in $l_{1}$ and $l_{2}$.

A3 Dominance Regions: $u_{t}(\theta, 1,1)<0$ for all $\theta<\underline{\theta}+3 \sigma$ and $u_{t}(\theta, 0,0)>0$ for all $\theta>\bar{\theta}-3 \sigma$.

Based on A1, we can interpret the fundamental $\theta$ as a quality of the project: higher fundamentals are associated with higher payoffs. Assumption A2 imposes strategic complementarities: investing by any agent in any round increases the incentive to invest for other agents in both rounds. Assumption A3 assures that in both stages of the dynamic and the static game, agents with very high signals participate in the project and those with very low signals do not participate. ${ }^{3,4}$

Agents have heterogeneous private information: Nature draws the (common) fundamental $\theta$ from a uniform distribution on $\Theta=[\underline{\theta}, \bar{\theta}] \subset \mathbb{R}$. We discuss the role of the uniform prior in Section 7 and conjecture that in the limit of precise signals our results remain valid under a non-uniform prior. At the beginning of rounds $t=1,2$, each agent $i$ observes a private signal

$$
x_{t}^{i}=\theta+\sigma \eta_{t}^{i} .
$$

\footnotetext{
${ }^{2}$ The results can be extended to allow for isolated payoff discontinuities such as those used in games of regime change. Static games of regime change are used to model currency attacks (Morris and Shin 1998), bank runs (Goldstein and Pauzner 2005) or revolutions (Edmond 2008). The attack in these models succeeds if its size exceeds a critical level.

${ }^{3}$ Assumption A3 can be weakened. See the working version of this paper, Kováč and Steiner (2008).

${ }^{4}$ For some results (in Section 5) we consider $\sigma \rightarrow 0$. Note that if Assumption A3 holds for some $\bar{\sigma}>0$, then it also holds for all $\sigma \in(0, \bar{\sigma})$.
} 
The vector of errors $\left(\eta_{1}^{i}, \eta_{2}^{i}\right)$ is i.i.d. across agents, independent of $\theta$ and distributed according to a continuous joint distribution with a compact convex support $H \subseteq[-1,1]^{2}$. The errors are not required to be independent across rounds, though errors independent across time are a special case of the setting. ${ }^{5}$ Opponents' actions are unobserved, but we can reinterpret the exogenous signals $x_{2}^{i}$ in round 2 in terms of social learning, see Section 7. There is no aggregate uncertainty: the realized population of errors is identical to their joint density.

Bold letter $\mathbf{x}^{i}=\left(x_{1}^{i}, x_{2}^{i}\right)$ denotes the type (signal pair) of agent $i$. The type set is

$$
X=\left\{\left(x_{1}, x_{2}\right): \exists \theta \in \mathbb{R} \text { and } \exists\left(\eta_{1}, \eta_{2}\right) \in H \text { such that }\left(x_{1}, x_{2}\right)=\left(\theta+\sigma \eta_{1}, \theta+\sigma \eta_{2}\right)\right\} ;
$$

it is the union of all diagonal translations of the error support $H$ scaled by $\sigma$, see Figure 2. Notice that the type set $X$ is larger than the support of types, as $X$ contains also types derived from $\theta \in \mathbb{R} \backslash \Theta$. With these redundant types included, the type set $X$ is translation-invariant, which will simplify discussion and notation. We use the usual incomplete product order $\leq$ to compare the types. A (pure) strategy is a pair of functions $\mathbf{s}=\left(s_{1}, s_{2}\right)$ with $s_{1}: \mathbb{R} \longrightarrow\{0,1\}, s_{2}: X \longrightarrow\{0,1\}$. Abusing terminology and notation, we also call signal $x_{1}^{i}$ in round 1 a type, and action rule $s_{1}\left(x_{1}^{i}\right)$ in round 1 a strategy.

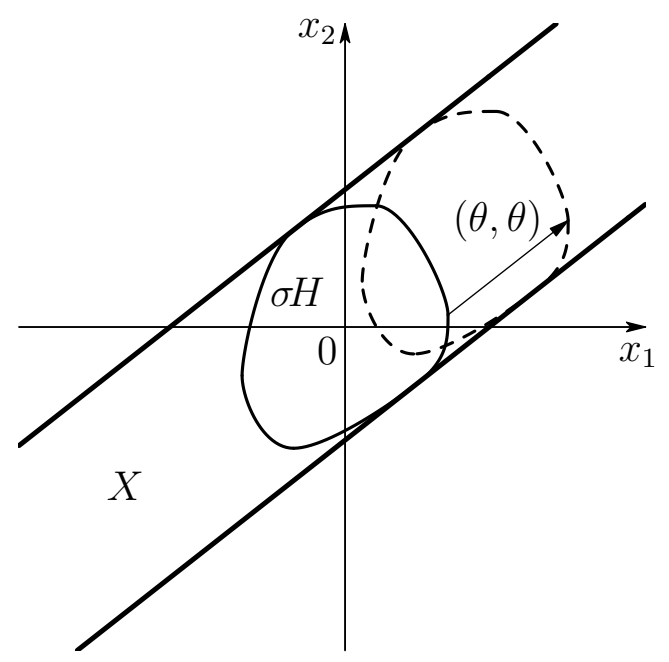

Figure 2: Support of errors and the type space.

Our main applied result, presented in Section 5, characterizes the effect of the reversibility option on the coordination outcome. To that end we compare the above dynamic game $\Gamma_{d y n}$ with a benchmark static game $\Gamma_{s t}$ which differs from $\Gamma_{d y n}$ only in the lack of the reversibility option: each agent can move only in round 1; once an agent invests in round 1 , she must stay in the project in round 2; see Figure 1. To facilitate the comparison with the dynamic game, we keep the lower index 1 when describing the signal

\footnotetext{
${ }^{5}$ In Section 5 we further restrict error distribution, ruling out independent errors. There we require that the first signal is an uninformative coarsening of the second signal.
} 
$x_{1}^{i}$ or strategy $s_{1}\left(x_{1}^{i}\right)$ in the static game despite it having only one non-trivial round.

\section{The Laplacian Property}

We now discuss how the model captures strategic uncertainty. Consider a symmetric, monotone strategy profile under which an agent in round 1 enters if and only if her signal $x_{1}^{i}$ exceeds a threshold $x_{1}^{*}$. We analyze the beliefs and expectations of the threshold type $x_{1}^{*}$. This type, being uncertain about the actions of others as well as about her own future action, embodies strategic uncertainty of the economic agent in the midst of a regime change. Moreover, understanding beliefs and expectations of the threshold type is essential for the equilibrium characterization, as the analysis relies on the indifference condition imposed on the type $x_{1}^{*}$.

In the next subsection, we review Morris and Shin (2003), who have described the beliefs of the threshold type in a simultaneous-move game. Then, in Subsection 3.2, we generalize the Laplacian property to the dynamic game under assumptions P1-3 imposed on endogenous objects. Later on, in Section 4, we prove that the assumptions A1-3, imposed on exogenous objects, imply that P1-3 are satisfied in the unique equilibrium.

\subsection{Belief about the First-round Aggregate Action}

Let $s_{1}\left(x_{1}^{i}\right)$ be a monotone strategy in either the static or in the first round of the dynamic game. Denote the threshold signal by $x_{1}^{*}$, and let $L_{1}=\left\{\mathbf{x}: x_{1} \geq x_{1}^{*}\right\}$ be the set of types investing in round 1 . The symmetric strategy profile $s_{1}$ induces a non-decreasing function $l_{1}(\theta)$ specifying aggregate investment for each realization of $\theta$. In the absence of aggregate uncertainty, $l_{1}(\theta)$ is the probability that an agent $j$ invests:

$$
l_{1}(\theta)=\operatorname{Pr}\left(\mathbf{x}^{j} \in L_{1} \mid \theta\right)
$$

The following theorem describes belief of the threshold type about the first-round action:

Theorem 1 (Laplacian Property). (Morris and Shin 2003)

The threshold type has no information about the aggregate action: the distribution of the conditional random variable $l_{1}(\theta) \mid x_{1}^{*}$ is uniform on $[0,1]$.

For convenience, we provide the proof in the Appendix.

The intuition is as follows. The threshold type $x_{1}^{*}$, being on the boundary between the investing and the non-investing types, is uncertain about the realized proportion of agents to the right of the boundary (the investing types). This proportion is determined by the rank of her signal $x_{1}^{*}$ within the realized population of signals. The only information 
she receives is her own signal, which is, due to the uniform prior, entirely uninformative about her rank, and therefore about the aggregate investment. Thus, she form uniform beliefs about the aggregate action.

Before we focus on the dynamic setting, we briefly discuss how the Laplacian belief determines equilibrium behavior in the static game. Consider a specification with very precise signals, $\sigma$ being small. In this case, the agents know that the fundamental $\theta$ is close to their private signals $x_{1}^{i}$. In particular, the threshold type $x_{1}^{*}$ knows that $\theta \approx x_{1}^{*}$, and she has the uniform belief about the aggregate action. As the threshold type is indifferent across the two available actions, it satisfies:

$$
0=E\left[u_{1}\left(\theta, l_{1}, l_{1}\right)+u_{2}\left(\theta, l_{1}, l_{1}\right) \mid x_{1}^{*}\right] \approx \int_{0}^{1}\left(u_{1}\left(x_{1}^{*}, l_{1}, l_{1}\right)+u_{2}\left(x_{1}^{*}, l_{1}, l_{1}\right)\right) d l_{1},
$$

which (approximately) determines the unique monotone, symmetric equilibrium. See Section 4 for details.

\subsection{Laplacian Expectations in the Dynamic Game}

The belief about the first-round aggregate action does not fully determine the equilibrium of the dynamic game. To impose the indifference condition on the threshold type $x_{1}^{*}$, we need to examine her beliefs about the both stages of the project.

We fix a symmetric strategy profile $\mathbf{s}=\left(s_{1}, s_{2}\right)$, and impose the following properties:

P1 Diagonal Monotonicity: $\mathbf{s}(\mathbf{x}+(d, d))$ is non-decreasing in $d$.

P2 Extreme Behavior: $\mathbf{s}(\mathbf{x})=(0,0)$ for $\mathbf{x} \leq(\underline{\theta}+2 \sigma, \underline{\theta}+2 \sigma)$, and $\mathbf{s}(\mathbf{x})=(1,1)$ for $\mathbf{x} \geq(\bar{\theta}-2 \sigma, \bar{\theta}-2 \sigma)$.

As we will prove in Section 4, A1-3 imply that the two properties are satisfied in the unique equilibrium. We maintain notation $x_{1}^{*}$ for the threshold signal in round 1 , and let $L_{1}$ and $L_{2}=\left\{\mathbf{x}: s_{1}\left(x_{1}\right)=s_{2}(\mathbf{x})=1\right\}$ denote the sets of types that invest in the first and in both rounds, respectively; see Figure 3 for illustration. Symmetric strategy profile $\mathbf{s}$ induces a pair of investment profiles - non-decreasing functions $l_{t}(\theta)=\operatorname{Pr}\left(\mathbf{x}^{j} \in L_{t} \mid \theta\right)$ that specify the investment levels in rounds $t=1,2$ for each realization of $\theta$. Out of the triple of variables $\theta, l_{1}, l_{2}$ we can choose any one as the independent one and express the remaining two variables as its non-decreasing functions. We abuse the notation by denoting those functions $\theta\left(l_{t}\right), l_{1}\left(l_{2}\right)$, and $l_{2}\left(l_{1}\right) .6$

Our long-term aim is to find equilibrium threshold signal $x_{1}^{*}$ above which agents enter the project in the first round and below which they stay out. We will characterize $x_{1}^{*}$ only

\footnotetext{
${ }^{6}$ Both $l_{1}(\theta)$ and $l_{2}(\theta)$ are non-decreasing. Thus, their inverse functions may not be uniquely defined for countably many values of $l$. We can choose any value of $\theta$ from the preimage $\left\{\theta: l_{t}(\theta)=l\right\}$ without an impact on the result. This is because each type assigns 0 probability to any particular value of $l_{t}$.
} 

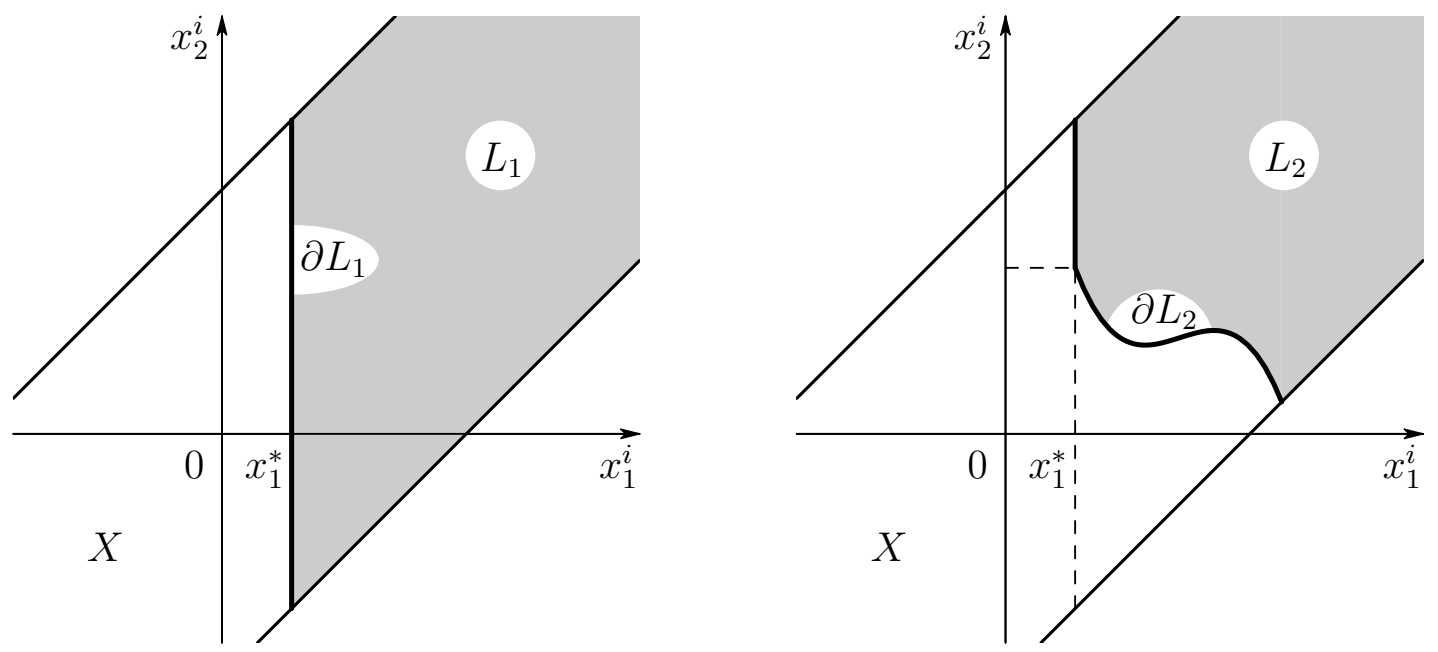

Figure 3: Illustration of the sets $L_{1}$ (types who enter in round 1) and $L_{2}$ (types who enter in round 1 and stay in round 2).

in the next section. In this section we focus on the payoff expectations of the threshold type.

We start by discussing behavior in the continuation game in round 2. We constrain for this section all agents to a possibly non-equilibrium, exogenous threshold $x_{1}^{*}$ in round 1 , and examine equilibrium of the continuation game: denote the expected continuation payoff of type $\mathbf{x}$ in round 2 by $U_{2}(\mathbf{x})=E\left[u_{2}\left(\theta, l_{1}, l_{2}\right) \mid \mathbf{x}\right]$, omitting from the notation that $U_{2}$ depends on the strategy $\mathbf{s}$ via mappings between $\theta, l_{1}$, and $l_{2}$. We impose a third property in this section that requires the agents to follow an equilibrium strategy of the continuation game:

P3 Optimality in the Continuation Play: $s_{2}(\mathbf{x})=1$ if and only if $U_{2}(\mathbf{x}) \geq 0$.

Let us now return to round 1 . We write $D_{t}$ for the expected payoff for stages $t=1,2$ as expected in round 1 by the threshold type $x_{1}^{*}:{ }^{7}$

$$
D_{1}=E\left[u_{1}\left(\theta, l_{1}, l_{2}\right) \mid x_{1}^{*}\right], \quad D_{2}=E\left[s_{2}\left(\mathbf{x}^{i}\right) \cdot U_{2}\left(\mathbf{x}^{i}\right) \mid x_{1}^{*}\right]
$$

Let us interpret the term $D_{2}$. Recall that $s_{2}\left(\mathbf{x}^{i}\right)=1$ when the agent stays in round 2 and $s_{2}\left(\mathbf{x}^{i}\right)=0$ when she exits in round 2 . In round 1 , the agent anticipates her own optimal behavior $s_{2}\left(\mathbf{x}^{i}\right)$ in round 2 , which is contingent on the yet unreceived signal $x_{2}^{i}$. Thus, $D_{2}$ is the value of the option to participate in the second stage, as evaluated in round 1.

The following theorem is the central technical insight of the paper. It states that the option value $D_{2}$ equals a simple payoff expectation of an agent who has no information about the aggregate action in round 2 , and who is committed to stay in the project:

\footnotetext{
${ }^{7}$ Again, the profile $\mathbf{s}$ and the mutual dependence of $\theta, l_{1}$, and $l_{2}$ are omitted from the notation.
} 


\section{Theorem 2 (Generalized Laplacian Property).}

Payoff expectations formed by threshold type $x_{1}^{*}$ in round 1 are equal to expectations formed under the uniform belief about $l_{1}$ and $l_{2}$, respectively:

$$
D_{1}=\int_{0}^{1} u_{1}\left(\theta\left(l_{1}\right), l_{1}, l_{2}\left(l_{1}\right)\right) d l_{1}, \quad D_{2}=\int_{0}^{1} u_{2}\left(\theta\left(l_{2}\right), l_{1}\left(l_{2}\right), l_{2}\right) d l_{2} .
$$

The expression for $D_{1}$ is an immediate consequence of Theorem 1, by which the threshold type $x_{1}^{*}$ in round 1 has the uniform belief about $l_{1}$. The characterization of $D_{2}$ is, however, not immediate. To see the intricacies, let us discuss a direct computation of the option value $D_{2}$.

The threshold type $x_{1}^{*}$ first computes the probability that she stays in the project upon receiving $x_{2}^{i}$, and then forms beliefs about aggregate actions conditional on staying. Such conditional beliefs are complicated as they reflects both the exogenous error distributions and the endogenous strategy profile. The advantage of Theorem 2 is that it circumvents the computation of such beliefs. The simple integral in (1), based on the uniform distribution of $l_{2}$ instead of the complicated conditional distribution, gives the correct value of $D_{2}$. The error distributions and the strategy profile still influence $D_{2}$ but they are summarized by the functions $\theta\left(l_{2}\right)$ and $l_{1}\left(l_{2}\right)$ that relate the fundamental and the investment levels in rounds 1 and 2 . Theorem 2 is useful despite that the functions $\theta\left(l_{2}\right), l_{1}\left(l_{2}\right)$ may be complicated, because the theorem will allow us to make predictions independent of these mappings; see Section 5.

We prove Theorem 2 in two auxiliary lemmas. In Lemma 1 we transform the agent's advantage arising from the exit option into an advantage arising from modified information. Transforming the original dynamic problem into a static one with the modified information is useful; known results on static global games do not accommodate our dynamic setting, but they are robust with respect to the information structure. Indeed, in Lemma 2, we recognize that the transformed problem is a static problem in which the known static Laplacian property, Theorem 1, holds. ${ }^{8}$

Before formulating the lemmas, we amend the notation. The new notation, though it is more complex, will highlight similarities between the terms $D_{1}$ and $D_{2}$. We say that $S \subset X$ is a diagonally monotone set, if it satisfies the following two properties: (i) if $\mathbf{x} \in S$, then $\mathbf{x}+(d, d) \in S$ for all $d \geq 0$. (ii) For any $\mathbf{x} \in X$ there exists $d \geq 0$ such that $\mathbf{x}-(d, d) \notin S$ and $\mathbf{x}+(d, d) \in S$. Notice that sets $L_{1}$ and $L_{2}$ are diagonally monotone by $\mathrm{P} 1-2$.

\footnotetext{
${ }^{8}$ Similar proof technique has been used in Sákovics and Steiner (2012) who analyze a static global game with heterogenous population. They map the original problem to a virtual static homogenous problem, achieving thus a partial characterization of the original strategic beliefs.
} 
We define (diagonal) boundary of a diagonally monotone set $S$ as

$$
\partial S=\{\mathbf{x} \in X: \mathbf{x}+(d, d) \in S \text { and } \mathbf{x}-(d, d) \notin S \text { for all } d>0\}
$$

Recall that $L_{1}$ is the set of types with $x_{1} \geq x_{1}^{*}$; see also Figure 3 . Therefore, the information of an agent who has received signal $x_{1}^{*}$ in round 1 is equivalent to the information that her type $\mathbf{x}$ belongs to the boundary $\partial L_{1}$.

We can rewrite $D_{1}$ and $D_{2}$ as

$$
D_{1}=E\left[u_{1}\left(\theta, l_{1}, l_{2}\right) \mid \partial L_{1}\right], \quad D_{2}=E\left[s_{2}\left(\mathbf{x}^{i}\right) \cdot U_{2}\left(\mathbf{x}^{i}\right) \mid \partial L_{1}\right]
$$

where we write $E[\xi \mid S]$ for the expectation of a random variable $\xi$ conditional on the event that type $\mathbf{x}^{i} \in S$, and define $E[\xi \mid \partial S]$ as $\lim _{d \rightarrow 0} E\left[\xi \mid \partial S^{d}\right]$, where $\partial S^{d}=\{\mathbf{x} \in X$ : $\mathbf{x}-\left(d^{\prime}, d^{\prime}\right) \in \partial S$ for some $\left.d^{\prime} \in[-d, d]\right\} .^{9}$

The first auxiliary lemma states that $D_{2}$ satisfies a formula analogous to the definition of $D_{1}$ :

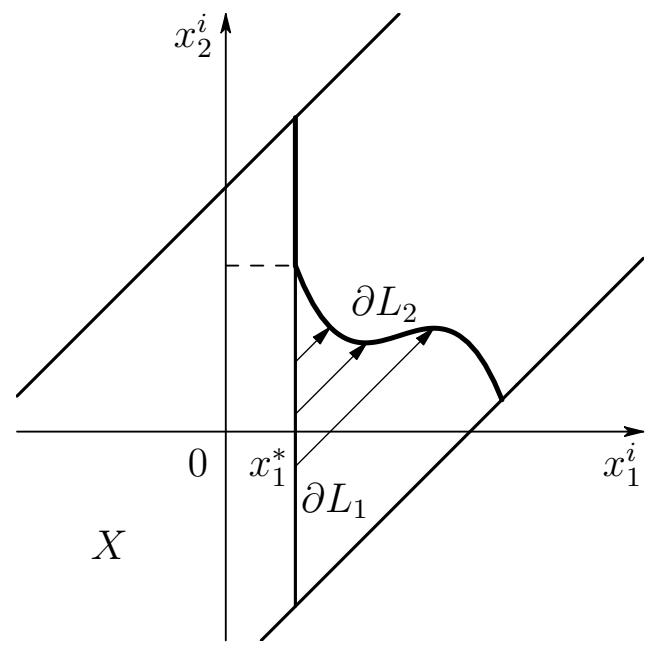

Figure 4: Lemma 1. Exiting types on $\partial L_{1}$ are replaced by indifferent types on $\partial L_{2}$.

Lemma 1. If strategy $\mathrm{s}$ satisfies P1-3 then

$$
D_{2}=E\left[u_{2}\left(\theta, l_{1}, l_{2}\right) \mid \partial L_{2}\right]
$$

The lemma expresses $D_{2}$ without the computational intricacies caused by the exit option. $D_{2}$ equals the expectation of an agent committed to stay in round 2, whose information is modified. Instead of the boundary of $L_{1}$, she conditions on being on the boundary of $L_{2}$. The lemma states that this change in information precisely compensates for the lost option.

\footnotetext{
${ }^{9}$ We assume that $S$ is such that $\partial S^{d}$ occurs with a positive probability for small enough $d$.
} 
The modification of agent's information is depicted in Figure 4: The exiting types on $\partial L_{1}$ are replaced by the investing but indifferent types on $\partial L_{2}$. Such replacement transforms the original option value in (2) into the payoff expectation conditional on $\partial L_{2}$ of an agent who never exits in round 2 - into (3). To see this, consider a type $\left(x_{1}^{*}, x_{2}\right)$. Whether this type invests in round 2 depends on her signal $x_{2}^{i}$ in round 2 . The investing types belong to the boundary $\partial L_{2}$. The types who exit receive 0 second stage payoff. Consider an exiting type $\left(x_{1}^{*}, x_{2}\right)$ and replace it by its diagonal translation $\tilde{\mathbf{x}}$ that lies in $\partial L_{2} ; \tilde{\mathbf{x}}=\left(x_{1}^{*}, x_{2}\right)+(d, d)$ such that $\tilde{\mathbf{x}} \in \partial L_{2}$. Type $\tilde{\mathbf{x}}$ satisfies the indifference condition in round 2 , and thus also receives the same expected payoff $U_{2}(\mathbf{x})=0$ (this is where the optimality of the continuation strategy is used in the argument). Therefore, the replacement preserves the payoff of the original exiting type. It remains to show that the relative probability of the unreplaced types is the same for the both boundaries $L_{1}$ and $L_{2}$. We show in the Appendix that this follows from a translational symmetry of the assumed signal distribution.

Consider a diagonally monotone set $S$, and let $l_{S}(\theta)=\operatorname{Pr}\left(\mathbf{x}^{j} \in S \mid \theta\right)$ generalize the investment profile functions $l_{t}(\theta)$. The following lemma is a generalization of the static Laplacian property; for $S=L_{1}$ it replicates Theorem 1.

Lemma 2. Let $S$ be a diagonally monotone set. Then $l_{S}(\theta) \mid \partial S$ is uniformly distributed on $[0,1]$.

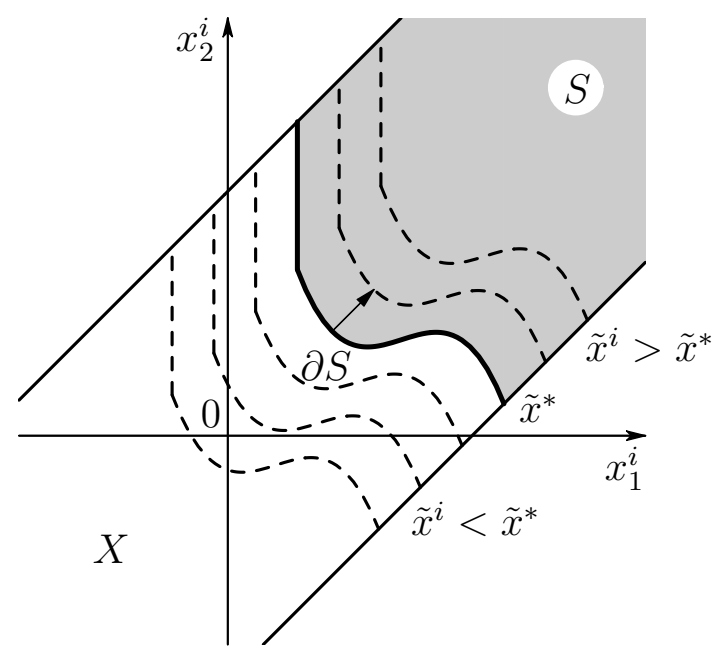

Figure 5: Lemma 2. Diagonal translations of $\partial S$. The information that an agent's type is on the boundary of $S$ is uninformative of the realized proportion of agents above the boundary.

Recall the intuition from the static game. Again, the information that the agent's type is somewhere on the boundary of $S$ is entirely uninformative of the realized proportion $l_{S}(\theta)$ of agents above the boundary. To show this formally, we reduce the dimensionality of the problem in the proof. We introduce diagonal translations of $\partial S$ and label them in 
a monotonic way as illustrated in Figure $5 .{ }^{10}$ We call the labels virtual one-dimensional signals $\tilde{x}^{i}$, and denote the label of $\partial S$ by $\tilde{x}^{*}$. Belief $l_{S}(\theta) \mid \partial S$ is then identical to the belief of the virtual threshold type $\tilde{x}^{*}$ about the measure of agents with $\tilde{x}^{i} \geq \tilde{x}^{*}$. It is the strategic belief of the threshold type in the static setting — the static Laplacian property, Theorem 1, applies.

Having established Lemmas 1 and 2, the proof of Theorem 2 becomes straightforward.

Proof of Theorem 2. By Lemma 1, $D_{t}=E\left[u_{t}\left(\theta, l_{1}, l_{2}\right) \mid \partial L_{t}\right]$. Lemma 2 with $S$ specified as $L_{t}$ gives that $l_{t} \mid \partial L_{t}$ is uniformly distributed on $[0,1]$. Combined, this implies generalized Laplacian property (1).

\section{Symmetric Monotone Equilibrium}

Before proceeding to the dynamic game, we first review the result in Morris and Shin (2003) for the static game. For each candidate $x_{1}^{*} \in \mathbb{R}$ for the equilibrium threshold, consider type $x_{1}^{*}$ 's incentive to invest. That is, consider symmetric monotone strategy profile $s_{1}$ with the threshold $x_{1}^{*}$, and for $t=1,2$ let

$$
S_{t}\left(x_{1}^{*}\right)=E\left[u_{t}\left(\theta, l_{1}, l_{1}\right) \mid x_{1}^{*}\right]
$$

be the expectations of the stage- $t$ payoff formed by the threshold type $x_{1}^{*}$ in the static game. We say that $x^{*} \in \mathbb{R}$ is a root of a function $m: \mathbb{R} \longrightarrow \mathbb{R}$, if $m(x)<0$ for $x<x^{*}$ and $m(x)>0$ for $x>x^{*}$ for $x$ in some neighborhood of $x^{*} \cdot{ }^{11}$ The equilibrium value of the threshold type is the root of the function $m_{s t}$ that denotes the threshold type's incentive to invest,

$$
m_{s t}\left(x_{1}^{*}\right)=S_{1}\left(x_{1}^{*}\right)+S_{2}\left(x_{1}^{*}\right) .
$$

By the state monotonicity A1, the function $m_{s t}$ is increasing, and the dominance regions assure that it attains both positive and negative values. Thus, $m_{s t}$ has a root and the static game has a unique monotone, symmetric Bayes-Nash equilibrium.

Let us now overview the analysis in the dynamic setting. Consider a symmetric strategy profile $\left(s_{1}, s_{2}\right)$ where $s_{1}$ is a threshold strategy with the threshold $x_{1}^{*}$. Similar to the function $m_{s t}$, we define

$$
m_{\text {dyn }}\left(x_{1}^{*}\right)=D_{1}\left(x_{1}^{*}\right)+D_{2}\left(x_{1}^{*}\right)
$$

\footnotetext{
${ }^{10}$ As $S$ is diagonally monotone, set of all diagonal translations of $\partial S$ form a partition of $X$.

${ }^{11}$ We use the definition of a root in this generalized sense, as below we work with functions for which we have established strict monotonicity but not continuity. If such a function attains both positive and negative values, the equation $m(x)=0$ may not have a solution, but it has a unique root in the sense defined above.
} 
to be the payoff expectation of the threshold type $x_{1}^{*}$ in round 1 of the dynamic game under the profile s. Again, the equilibrium threshold type $x_{1}^{*}$ is the root of $m_{d y n}$. To define $m_{d y n}$ : $\mathbb{R} \longrightarrow \mathbb{R}$ as an unambiguous function of its argument $x_{1}^{*}$, we specify the continuation strategy $s_{2}$ as a function of $x_{1}^{*}$. It is an equilibrium strategy in the continuation game: for each value of the threshold $x_{1}^{*}$, we consider the continuation game $\Gamma_{2}\left(x_{1}^{*}\right)$ induced from $\Gamma_{d y n}$ by the first round threshold $x_{1}^{*}$; agents must follow threshold $x_{1}^{*}$ in round 1 of $\Gamma_{2}\left(x_{1}^{*}\right)$ and only choose the continuation strategy $s_{2}\left(\mathbf{x}^{i}\right)$. Thus, $\Gamma_{2}\left(x_{1}^{*}\right)$ is a static Bayesian game. We prove in Appendix that $\Gamma_{2}\left(x_{1}^{*}\right)$ has a unique equilibrium for each value of $x_{1}^{*}$. Additionally, we prove the monotonicity of $m_{d y n}$.

Lemma 3. Function $m_{d y n}\left(x_{1}^{*}\right)$ is well-defined, increasing, and has a unique root.

The unique root of $m_{d y n}\left(x_{1}^{*}\right)$ and the induced continuation strategy $s_{2}$ constitute the unique symmetric monotone equilibrium of the dynamic game. The following proposition proposition summarizes the results. ${ }^{12}$

Proposition 1. The dynamic game has a unique symmetric monotone Bayes-Nash equilibrium. The equilibrium threshold in the first round, $x_{1}^{*}$, is the unique root of the function $m_{\text {dyn }}$. The equilibrium strategy satisfies properties P1-3.

\section{$5 \quad$ Strategic Effects of Reversibility}

In this section we return to our applied question. We characterize the strategic effects of the exit option by comparing equilibrium behavior across the dynamic and the static game. We identify conditions under which the option hampers or enhances coordination on the risky investment.

We proceed by comparing functions $m_{s t}$ and $m_{d y n}$. Consider, for instance, a situation as on Figure 6 , with $m_{d y n}\left(x_{1}^{*}\right) \geq m_{s t}\left(x_{1}^{*}\right)$ for all $x_{1}^{*}$. Then entry is an equilibrium action in round 1 on a larger set of types $x_{1}$ in the dynamic game than in the static game the exit option enhances coordination on entry.

The comparison of the two functions,

$$
m_{s t}\left(x_{1}^{*}\right)=S_{1}\left(x_{1}^{*}\right)+S_{2}\left(x_{1}^{*}\right) \quad \text { and } \quad m_{d y n}\left(x_{1}^{*}\right)=D_{1}\left(x_{1}^{*}\right)+D_{2}\left(x_{1}^{*}\right),
$$

would be difficult to establish by a direct computation as both the beliefs and the aggregate actions differ across the static and the dynamic game. We conduct the comparison using the Laplacian property; it assures that the expectations of the threshold types are based on the uniform beliefs in both games. The property allows for an unambiguous comparison of the terms $S_{t}$ and $D_{t}$ :

\footnotetext{
${ }^{12}$ Proposition 1 can be strengthened. As we show in the working paper Kováč and Steiner (2008), the dynamic game is dominance solvable, so no other, non-monotone or assymetric equilibria exist.
} 


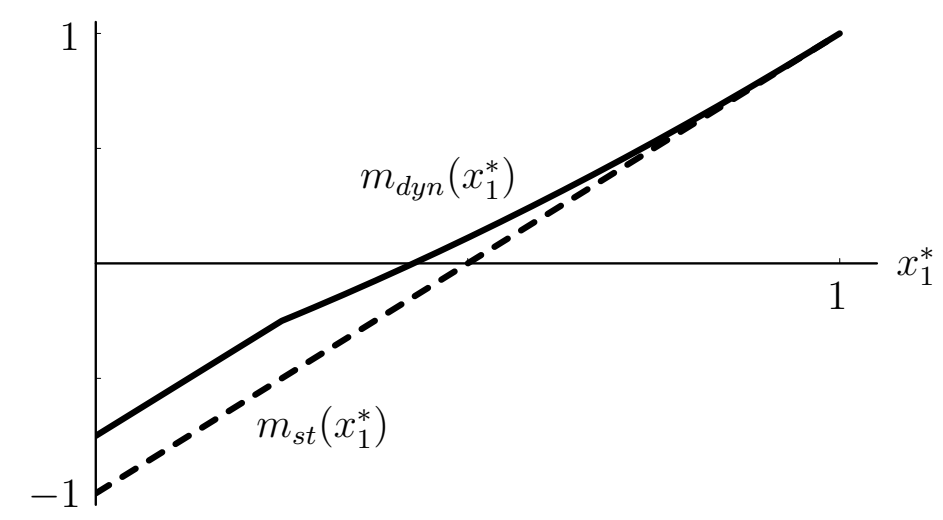

Figure 6: Comparison of functions $m_{s t}\left(x_{1}^{*}\right)$ and $m_{d y n}\left(x_{1}^{*}\right)$.

Lemma 4. For each $x_{1}^{*} \in \mathbb{R}$, the following inequalities hold:

(i) $S_{1}\left(x_{1}^{*}\right) \geq D_{1}\left(x_{1}^{*}\right)$,

(ii) $S_{2}\left(x_{1}^{*}\right) \leq D_{2}\left(x_{1}^{*}\right)$.

Proof. (i) Using the Laplacian property (Theorem 1) we can write both sides as expectations based on the uniform belief about $l_{1}$ :

$$
S_{1}\left(x_{1}^{*}\right)=\int_{0}^{1} u_{1}\left(\theta\left(l_{1}\right), l_{1}, l_{1}\right) d l_{1} \geq \int_{0}^{1} u_{1}\left(\theta\left(l_{1}\right), l_{1}, l_{2}\left(l_{1}\right)\right) d l_{1}=D_{1}\left(x_{1}^{*}\right) .
$$

The two integrals differ only in the third argument of $u_{1} \cdot{ }^{13}$ The inequality follows from the monotonicity of $u_{1}$ and from the fact that $l_{1} \geq l_{2}$. The last inequality is a technological constraint reflecting that agents can exit the project but cannot join it in the second round.

(ii) Similarly, by Theorem 2, we can write both sides as expectations based on the uniform belief about $l_{2}$ :

$$
S_{2}\left(x_{1}^{*}\right)=\int_{0}^{1} u_{2}\left(\theta^{s t}\left(l_{2}\right), l_{2}, l_{2}\right) d l_{2} \leq \int_{0}^{1} u_{2}\left(\theta^{d y n}\left(l_{2}\right), l_{1}\left(l_{2}\right), l_{2}\right) d l_{2}=D_{2}\left(x_{1}^{*}\right) .
$$

In this case, the two integrals differ in the first and second argument of $u_{2}$. For the second argument, we again use the inequality $l_{2} \leq l_{1}$ and the monotonicity of $u_{2}$. For the first argument, we use the monotonicity of $u_{2}$ with respect to $\theta$, and inequality $\theta^{\text {st }}\left(l_{2}\right) \leq$ $\theta^{d y n}\left(l_{2}\right)$. The last inequality is established as follows. The functions $\theta^{s t}\left(l_{2}\right)$ and $\theta^{d y n}\left(l_{2}\right)$ are defined as inverse functions to $l_{1}(\theta)=\operatorname{Pr}\left(\mathbf{x}^{i} \in L_{1} \mid \theta\right)$ and $l_{2}(\theta)=\operatorname{Pr}\left(\mathbf{x}^{i} \in L_{2} \mid \theta\right)$, respectively. The two functions are non-decreasing and, as $L_{1} \supseteq L_{2}, l_{1}(\theta) \geq l_{2}(\theta)$. Thus, the opposite inequality holds for the inverse functions.

\footnotetext{
${ }^{13}$ Function $l_{1}(\theta)=\operatorname{Pr}\left(x_{1}^{i} \geq x_{1}^{*} \mid \theta\right)$ in both games. Thus, the inverse function $\theta\left(l_{1}\right)$ is identical across the two games as well.
} 
We call inequalities (i) and (ii) the first and the second stage effects. The two effects are of opposite signs and therefore a comparison of $m_{s t}$ and $m_{d y n}$ is possible only if we impose further structure on the model:

Definition 1. We say that the payoffs do not exhibit

1. backward spillovers if $u_{1}\left(\theta, l_{1}, l_{2}\right)$ does not depend on $l_{2}$.

2. forward spillovers if $u_{2}\left(\theta, l_{1}, l_{2}\right)$ does not depend on $l_{1}$.

The absence of either backward or forward spillovers allows to characterize the strategic effect of reversibility. Assume first that payoffs do not exhibit backward spillovers. Then the first stage effect disappears, ${ }^{14}$

$$
S_{1}\left(x_{1}^{*}\right)=\int_{0}^{1} u_{1}\left(\theta\left(l_{1}\right), l_{1}\right) d l_{1}=D_{1}\left(x_{1}^{*}\right)
$$

and thus $m_{d y n}\left(x_{1}^{*}\right) \geq m_{s t}\left(x_{1}^{*}\right)$ by the second stage effect, as on Figure 6 . Therefore, in the absence of backward spillovers, the exit option enhances entry: agents enter in the dynamic game on a larger set of first-round signals $x_{1}$ than in the static game.

The result is reversed if payoffs do not exhibit forward spillovers. Let us explain the argument informally in the limit of small errors. When $\sigma$ is small then $x_{1} \approx \theta$. In particular the threshold type knows that $\theta$ is close to $x_{1}^{*}$. Thus:

$$
S_{2}\left(x_{1}^{*}\right) \approx \int_{0}^{1} u_{2}\left(x_{1}^{*}, l_{2}\right) d l_{2} \approx D_{2}\left(x_{1}^{*}\right) .
$$

Therefore, $m_{d y n}\left(x_{1}^{*}\right) \leq m_{s t}\left(x_{1}^{*}\right)$ by the first stage effect. Thus, the exit option hampers coordination on entry. In this case, though the exit option allows agents to react to the arriving information, this valuable flexibility is exceeded by the fear of a panic-based exit wave.

We will formalize these observations in the limit of precise signals, $\sigma \rightarrow 0$. In such a limit, the fundamental uncertainty becomes negligible, $x_{1}^{i} \approx x_{2}^{i} \approx \theta$, and the analysis can focus on the strategic uncertainty of the threshold type, which remains large. Formally, we examine sequences of the static and the dynamic games with varying scale of noise $\sigma$. The corresponding games are denoted by $\Gamma_{s t}(\sigma)$ and $\Gamma_{d y n}(\sigma)$, whereas $\Gamma_{s t}$ and $\Gamma_{d y n}$ without the argument denote in this section classes of games $(\Gamma(\sigma))_{\sigma}$. We now introduce a new terminology that describes local equilibrium behavior in the limit of precise signals.

Definition 2. We say that action path $h \in\{0,10,11\}$ is selected at $\theta$ in $\Gamma_{s t}$, respectively in $\Gamma_{d y n}$ if there exists $\bar{\sigma}>0$ such that for all $\sigma \in(0, \bar{\sigma}]$ all agents in $\Gamma_{s t}(\sigma)$, respectively in $\Gamma_{d y n}(\sigma)$, reach action path $h$ whenever Nature draws the fundamental $\theta$ and all agents follow the unique monotone, symmetric equilibrium profile.

\footnotetext{
${ }^{14}$ When $u_{t}$ does not depend on $l_{t^{\prime}}$, we drop the relevant argument.
} 
Naturally, only action histories 0 or 11 can be selected in the static game.

The next definition will allow us to focus on cases in which the second-round behavior is non-trivial.

Definition 3. We say that strategic uncertainty permits investment at $\theta$ in round 2 if

$$
\int_{0}^{1} u_{2}\left(\theta, 1, l_{2}\right) d l_{2}>0
$$

The opposite inequality trivially rules out investment in round 2. To see this, assume that $\int_{0}^{1} u_{2}\left(\theta, 1, l_{2}\right) d l_{2}<0$. We will show that agents cannot coordinate on staying in the project even under the best possible scenario. The intuition is as follows. To make the second stage as attractive as possible, suppose that the first stage of the project is compulsory, $l_{1}=1$. The game, effectively collapsing to the second stage decisions, becomes a static game. Given the assumed inequality, action 0 is selected in this static global game at $\theta$. Let us now return to the setting with voluntary investment in round 1 . When $\int_{0}^{1} u_{2}\left(\theta, 1, l_{2}\right) d l_{2}<0$, all agents expect in round 1 of the dynamic game that they will exit in round 2 . Thus, the dynamic game essentially simplifies into a static game consisting of only the first round. As analysis of the exit option then reduces to a simple comparison of two static global games, we omit such cases.

We impose two additional, technical assumptions:

A4 Strict Action Monotonicity in Round 2: $u_{2}\left(\theta, l_{1}, l_{2}\right)$ is increasing in $l_{2}$.

A5 Restriction on Error Distributions: $\eta_{1}^{i}-\eta_{2}^{i}$ is independent of $\eta_{2}^{i}$.

Assumption A5 ensures that the first signal $x_{1}^{i}$ is an uninformative coarsening of $x_{2}^{i},{ }^{15}$ and thus, $x_{2}^{i}$ is sufficient statistics for $\theta$ given $\left(x_{1}^{i}, x_{2}^{i}\right) .{ }^{16}$ Thus the optimal strategy in round 2 depends only on $x_{2}^{i}$ and not on $x_{1}^{i}$. One-dimensionality of the continuation strategy simplifies the proofs of the limit results below, but we conjecture that the results hold for a large family of other error structures as well.

We are now ready to state the main applied results. The exit option enhances coordination on investment in the absence of backward spillovers. The effect is reversed when payoffs do not exhibit forward spillovers. Assume for the next two propositions and for the corollary that strategic uncertainty permits investment at $\theta$ in round 2 .

Proposition 2. If payoffs do not exhibit backward spillovers, then the exit option enhances investment:

(i) If action path 11 is selected at $\theta$ in $\Gamma_{\text {st }}$, then 11 is selected at $\theta$ in $\Gamma_{d y n}$.

\footnotetext{
${ }^{15}$ Recall that $x_{1}^{i}=x_{2}^{i}+\left(\eta_{1}^{i}-\eta_{2}^{i}\right)$.

${ }^{16}$ Assumption A5 is also used in the existing literature (see, for example, Heidhues and Melissas 2006).
} 
(ii) If 0 is selected at $\theta$ in $\Gamma_{d y n}$, then 0 is selected at $\theta$ in $\Gamma_{s t}$.

Proposition 3. If payoffs do not exhibit forward spillovers, then the exit option hampers investment:

(i) If action path 11 is selected at $\theta$ in $\Gamma_{d y n}$, then 11 is selected at $\theta$ in $\Gamma_{\text {st }}$.

(ii) If 0 is selected at $\theta$ in $\Gamma_{\text {st }}$, then 0 is selected at $\theta$ in $\Gamma_{d y n}$.

Let us remark that Proposition 2 can be extended to settings with any $\sigma>0$, whereas the result in Proposition 3 holds only for the vanishing $\sigma$. This is because Proposition 2 is based on (6) which holds for any $\sigma$, while Proposition 3 is driven by (7) that holds only for vanishing $\sigma$.

A simple corollary of the last two propositions is that in the absence of both spillovers, the provision of the exit option does not change the coordination outcome.

Corollary 1. If payoffs do not exhibit forward nor backward spillovers, then the same action path is selected at $\theta$ in $\Gamma_{s t}$ and $\Gamma_{d y n}$.

\section{$5.1 \quad$ Example}

We use an example to illustrates two seemingly contradicting features of the model. (i) The strategic effect of the exit option is typically large - the option changes equilibrium behavior with positive ex ante probability even for vanishing $\sigma$. Yet, (ii) the direct effect of the exit option is negligible - the ex ante probability that the option is used vanishes as $\sigma \rightarrow 0$.

Consider an information structure where, consistently with Assumption A5, $x_{1}^{i}=$ $\theta+\sigma \eta_{2}^{i}+\sigma \eta_{\Delta}^{i}$ and $x_{2}^{i}=\theta+\sigma \eta_{2}^{i}$, where $\eta_{\Delta}^{i}$ and $\eta_{2}^{i}$ are independent. Let the distribution of $\eta_{\Delta}^{i}$ be uniform on $[-1,1]$, and the distribution of $\eta_{2}^{i}$ uniform on $[-\varepsilon, \varepsilon]$, where $0<\varepsilon \ll 1$. The payoff functions are

$$
u_{1}\left(\theta, l_{1}, l_{2}\right)=\theta+l_{1}, \quad u_{2}\left(\theta, l_{1}, l_{2}\right)=\theta+\frac{l_{1}+l_{2}}{2} .
$$

These payoffs do not exhibit backward spillovers and so, by Proposition 2, the exit option enhances investment. Thus, we can conclude without detailed computations that, in this case, entry is selected on a weakly larger set of $\theta$ in the dynamic than in the static game. Detailed computation reveals that the effect is strict. That is, as $\sigma \rightarrow 0$, entry is selected on a strictly larger set of $\theta$ in the dynamic than in the static game.

The two depicted functions in Figure 6 illustrate $m_{d y n}$ and $m_{s t}$ for this particular specification. Their computation is based on the first- and the second-round indifference conditions imposed on thresholds $x_{1}^{*}$ and $x_{2}^{*}$. The two thresholds specify the mappings between $\theta, l_{1}$ and $l_{2}$, and the mappings, together with the Laplacian property, in turn 
fully specify the functions $m_{s t}$ and $m_{d y n}$. See Kováč and Steiner (2008) for detailed computation of $m_{s t}$ and $m_{d y n}$.

We first discuss the feature (i). The functions $m_{d y n}$ and $m_{s t}$ are depicted in Figure 6 in the limit, as $\sigma \rightarrow 0$. The difference between the roots of the two functions does not disappear in the limit. Thus, the strategic consequence of the exit option is large in the sense, that the interval of $\theta$ at which entry is selected in the dynamic but not in the static game has a positive ex ante probability.

Let us now discuss (ii). We have verified for this specification that strategic uncertainty permits investment at $\theta$ in round 2 for all $\theta$ above the root of $m_{d y n}$. We show in the Appendix (Lemma 9) that in such a case entry followed by exit is never selected. For all $\theta$ above the root $x_{1}^{*}$ of $m_{d y n}$ entry and staying in the project is selected, while for $\theta$ below the root not investing is selected.

To reconcile (i) and (ii), recall that a player with the first-round signal $x_{1}^{*}$ exercises the exit option with a positive probability, because she suffers from large strategic uncertainty and her beliefs may shift from optimistic to pessimistic in between the rounds 1 and 2 . While exiting types are rare ex ante, the exit option has a large strategic impact. This is because the pivotal equilibrium condition - the indifference condition - is imposed on the threshold type $x_{1}^{*}$, and probability of exiting, conditional on $x_{1}^{i}=x_{1}^{*}$, is non-vanishing as $\sigma \rightarrow 0$.

Why does the exit option enhance investment in this case? The first-stage effect disappears, as the type $x_{1}^{*}$ has an identical belief about $l_{1}$ across the both games, and $u_{1}$ does not depend on $l_{2}$. The second stage effect is non-vanishing; the type $x_{1}^{*}$ is (precisely, behaves as if it was) more optimistic about $l_{1}$ in the dynamic than in the static game. In both games, the type $x_{1}^{*}$ "has" uniform belief about $l_{2}$, but in the static game $l_{1}=l_{2}$, while in the dynamic game $l_{1}>l_{2}$.

\section{Extension: Laplacian Property for Many Rounds}

This section generalizes the Laplacian Property to more than two rounds. Following the description of the model in Section 2, consider an investment project with $T \in \mathbb{N}$ production stages and agents having the option to exit before every stage. The agent who exists in round $t=2, \ldots, T+1$ obtains a payoff

$$
\sum_{\tau=1}^{t-1} u_{\tau}\left(\theta, l_{1}, l_{2}, \ldots, l_{T}\right)
$$


where $l_{t}$ is the measure of agents who are still active after round $t .{ }^{17}$ The agent who does not participate in the project at all (exists in round 1) obtains a payoff 0.

At the beginning of each round $t=1,2, \ldots, T$, before making the exit/stay decision, every agent $i$ observes a private signal $x_{t}^{i}=\theta+\sigma \eta_{t}^{i}$ with errors having the same properties as in Section 2. The type set $X \subseteq \mathbb{R}^{T}$ is, as in the baseline model, the union of all diagonal translations of the error support (scaled by $\sigma$ ). Strategy is a vector of functions $\mathbf{s}=\left(s_{1}, \ldots, s_{T}\right)$, where $s_{t}: X \longrightarrow\{0,1\}$ depends only on signals $x_{1}, \ldots, x_{t}$.

Let $L_{t}=\left\{\mathbf{x}: s_{\tau}\left(x_{1}, \ldots, x_{\tau}\right)=1\right.$ for all $\left.\tau=1, \ldots, t\right\}$ and $l_{t}(\theta)=\operatorname{Pr}\left(\mathbf{x}^{j} \in L_{t} \mid \theta\right)$. Similarly, as in Section 3.2, variables $\theta, l_{1}, \ldots, l_{T}$ are mutually dependent and any of them can be chosen as independent variable determining other $T$ variables. As before, $U_{t}(\mathbf{x})=$ $E\left[u_{t}\left(\theta, l_{1}, \ldots, l_{T}\right) \mid x_{1}, \ldots, x_{t}\right]$ is the expected payoff of type $\mathbf{x}$ in round $t$, conditional on her information at $t$.

We again consider a symmetric strategy profile that satisfies the properties of Diagonal Monotonicity P1, Extreme Behavior P2, and Optimality in the Continuation Play P3. ${ }^{18}$ To define the last property, we introduce a value function $V_{t}(\mathbf{x})$ by $V_{T+1}=0$, and

$$
V_{t}(\mathbf{x})=U_{t}(\mathbf{x})+s_{t+1}(\mathbf{x}) \cdot V_{t+1}(\mathbf{x}) \quad \text { for all } t=T, \ldots, 1 .
$$

The property P3 requires that $s_{t}(\mathbf{x})=1$ if and only if $V_{t}(\mathbf{x}) \geq 0$, for all $t \geq 2$.

Finally, let $D_{1}=E\left[u_{1} \mid x_{1}^{*}\right]$ and

$$
D_{t}=E\left[s_{2}(\mathbf{x}) \cdot \ldots \cdot s_{t}(\mathbf{x}) \cdot U_{t}(\mathbf{x}) \mid x_{1}^{*}\right]
$$

it is the expectation of the payoff for stage $t \geq 2$ as formed in round 1 by the threshold type $x_{1}^{*}$, taking into account the exit options.

The next theorem extends the generalized Laplacian Property to settings with multiple exit possibilities.

Theorem 3. Payoff expectations formed by threshold type $x_{1}^{*}$ in round 1 are equal to expectations formed under the uniform belief about $l_{t}$ :

$$
\sum_{t=1}^{T} D_{t}=\sum_{t=1}^{T} \int_{0}^{1} u_{t}\left(\theta\left(l_{t}\right), l_{1}\left(l_{t}\right), \ldots, l_{T}\left(l_{t}\right)\right) d l_{t}
$$

Proof of Theorem 3. The proof follows from Lemma 2 and Lemma 10, which is a generalization Lemma 1 (the statement and the proof of Lemma 10 is in Appendix A.5). As for

\footnotetext{
${ }^{17}$ To unify the terminology, if the agent stays in all rounds, we say that she exits in round $T+1$ with payoff of the above form.

${ }^{18}$ The properties P1 and P2 can be generalized in a straightforward manner. In equilibrium, the properties follow from assumptions analogous to Strict State Monotonicity A1, Weak Action Monotonicity A2, and Dominance Regions A3.
} 
Lemma 2, note that its proof is not restricted to 2-dimensional signals. It applies for any dimension with analogous definition of a diagonally monotone set and its boundary. ${ }^{19}$

Applying Lemma 10 to the value function, we obtain that for all $t=2, \ldots, T$ :

$$
E\left[s_{t}(\mathbf{x}) \cdot V_{t}(\mathbf{x}) \mid \partial L_{t-1}\right]=E\left[V_{t}(\mathbf{x}) \mid \partial L_{t}\right]
$$

Notice the relationship to Lemma 1: when $T=t=2$ the last equation gives $E\left[s_{1}(\mathbf{x})\right.$. $\left.U_{2}(\mathbf{x}) \mid \partial L_{1}\right]=E\left[U_{2}(\mathbf{x}) \mid \partial L_{2}\right]$ - the statement of Lemma 1.

By the Optimality in the Continuation Play P3, the left hand side of (8) equals $E\left[V_{1}(\mathbf{x}) \mid \partial L_{1}\right]$. This in turn equals the right-hand side of (8), as follows from the following decomposition:

$$
\begin{aligned}
E\left[V_{t}(\mathbf{x}) \mid \partial L_{t}\right] & =E\left[U_{t}(\mathbf{x}) \mid \partial L_{t}\right]+E\left[s_{t+1}(\mathbf{x}) \cdot V_{t+1}(\mathbf{x}) \mid \partial L_{t}\right] \\
& =E\left[U_{t}(\mathbf{x}) \mid \partial L_{t}\right]+E\left[V_{t+1}(\mathbf{x}) \mid \partial L_{t+1}\right]
\end{aligned}
$$

Applying this decomposition recursively for $t=1, \ldots, T-1$, we obtain $E\left[V_{1}(\mathbf{x}) \mid \partial L_{1}\right]=$ $\sum_{t=1}^{T} E\left[U_{t}(\mathbf{x}) \mid \partial L_{t}\right]$. This is indeed equal to the right-hand side of (8), as for each $t$ we have

$$
\begin{aligned}
E\left[U_{t}(\mathbf{x}) \mid \partial L_{t}\right] & =E\left[E\left[u_{t}\left(\theta, l_{1}, \ldots, l_{T}\right) \mid x_{1}, \ldots, x_{t}\right] \mid \partial L_{t}\right] \\
& =E\left[u_{t}\left(\theta, l_{1}, \ldots, l_{T}\right) \mid \partial L_{t}\right]=\int_{0}^{1} u_{t}\left(\theta\left(l_{t}\right), l_{1}\left(l_{t}\right), \ldots, l_{T}\left(l_{t}\right)\right) d l_{t},
\end{aligned}
$$

using the law of iterated expectations and Lemma 2.

The effects of reversibility described in Table 1 apply also in the setting with multiple rounds. We give a brief argument for this comparison, which is again based on monotonicity of the payoff functions and on the inequalities $l_{1} \geq l_{2} \geq \cdots \geq l_{T}$. We extend the notation by letting $S_{t}=E\left[u_{t}\left(\theta, l_{t}, \ldots, l_{t}\right) \mid x_{1}^{*}\right]=\int_{0}^{1} u_{t}\left(\theta^{s t}\left(l_{t}\right), l_{t}, \ldots, l_{t}\right) d l_{t}$ and we again use $m_{s t}=S_{1}+\cdots+S_{T}$ for the threshold investment incentive in the static game. As before, the payoffs do not exhibit backward spillovers if $u_{t}\left(\theta, l_{1}, \ldots, l_{T}\right)$ does not depend on $l_{t+1}, \ldots, l_{T}$ for all $t=1, \ldots, T-1$. The payoffs do not exhibit forward spillovers if $u_{t}\left(\theta, l_{1}, \ldots, l_{T}\right)$ does not depend on $l_{1}, \ldots, l_{t-1}$ for all $t=2, \ldots, T$. In the former case we obtain (dropping the irrelevant arguments):

$$
\int_{0}^{1} u_{t}\left(x_{1}^{*}, l_{1}\left(l_{t}\right), \ldots, l_{t-1}\left(l_{t}\right), l_{t}\right) d l_{t} \geq \int_{0}^{1} u_{t}\left(x_{1}^{*}, l_{t}, \ldots, l_{t}, l_{t}\right) d l_{t} .
$$

When $\sigma$ is small, this implies that $m_{d y n} \geq m_{s t}$. On the other hand, in the latter case we

\footnotetext{
${ }^{19} \mathrm{We}$ use the $T$-dimensional vector $(d, \ldots, d)$ instead of 2-dimensional $(d, d)$.
} 
have

$$
\int_{0}^{1} u_{t}\left(x_{1}^{*}, l_{t}\left(l_{t}\right), l_{t+1}, \ldots, l_{T}\left(l_{t}\right)\right) d l_{t} \leq \int_{0}^{1} u_{t}\left(x_{1}^{*}, l_{t}, \ldots, l_{t}, l_{t}\right) d l_{t} .
$$

Again, for $\sigma$ small we obtain that $m_{d y n} \leq m_{s t}$, leading to the same comparative results as in the setting with two rounds.

\section{$7 \quad$ Discussion of the model}

Let us briefly discuss the main modeling assumptions.

Uninformative prior. The uninformative prior together with the independence of errors with respect to $\theta$ imply that local properties of the information structure are independent of the realized value of $\theta$. That is, for two realizations of the fundamental $\theta$ and $\theta^{\prime}$, conditional density of $\mathbf{x} \mid \theta$ is a translation of the conditional density of $\mathbf{x} \mid \theta^{\prime}$. This translation invariance, which drives the Laplacian property, would be distorted by an informative prior. However, in the limit of small noise, as $\sigma \rightarrow 0$, any prior becomes approximately uninformative compared to informativeness of the signals. We conjecture our results remain valid under any prior in the limit of small noise. See Frankel, Morris and Pauzner (2003) for formalization of this idea for static global games.

Fixed fundamental. We assumed that the fundamental $\theta$ is fixed throughout the game. We abstract from the fluctuations in $\theta$ because learning alone suffices to induce positive value to the reversibility option, and the arguments behind the generalized Laplacian property are orthogonal to the fluctuations. We conjecture that the generalized Laplacian property remains valid in a randomly evolving environment.

Social learning. Let us discuss micro-foundations for our exogenous learning process in terms of social learning. The following modeling approach is often used in dynamic global games. ${ }^{20}$ In our baseline model, agents receive additional information about $\theta$ in round 2 , whereas the early investment level $l_{1}$ is unobserved. However, the agents can in equilibrium deduce additional information about $l_{1}$ from the signal $x_{2}^{i}$ : If all agents use a monotone strategy with threshold $x_{1}^{*}$ in round 1 , then $\theta$ and $l_{1}$ are related by the mapping $l_{1}=1-F_{1}\left(\frac{x_{1}^{*}-\theta}{\sigma}\right)$, where $F_{1}$ is the c.d.f. of the first-round error $\eta_{1}^{i}$.

We can reverse the perspective and formulate an alternative model in which the primary source of information in round 2 is a noisy observation of $l_{1}$ and agents deduce information about $\theta$ only indirectly. Assume in this alternative that agents in round 1 observe fundamental-based signal $x_{1}^{i}=\theta+\sigma \eta_{1}^{i}$ as in the baseline setup, but instead of the round 2 signal $x_{2}^{i}=\theta+\sigma \eta_{2}^{i}$, agents observe a noisy aggregate statistic of the round

\footnotetext{
${ }^{20}$ It was first used in Dasgupta (2007), and later used in Angeletos, Hellwig and Pavan (2007), Angeletos and Werning (2006), and in Goldstein, Ozdenoren and Yuan (2011).
} 
1 actions. The following specification is used in the literature for tractability reasons:

$$
y^{i}=1-F_{1}^{-1}\left(1-l_{1}\right)+\eta_{2}^{i} .
$$

In a symmetric monotone equilibrium, the observation of $y^{i}$ is equivalent to the observation of $x_{2}^{i}=\theta+\sigma \eta_{2}^{i}$ because an agent observing $y^{i}$ can compute $x_{2}^{i}$ in the equilibrium. Hence, the unique symmetric monotone equilibria coincide across our model with fundamental-based learning and the alternative model with social-based learning.

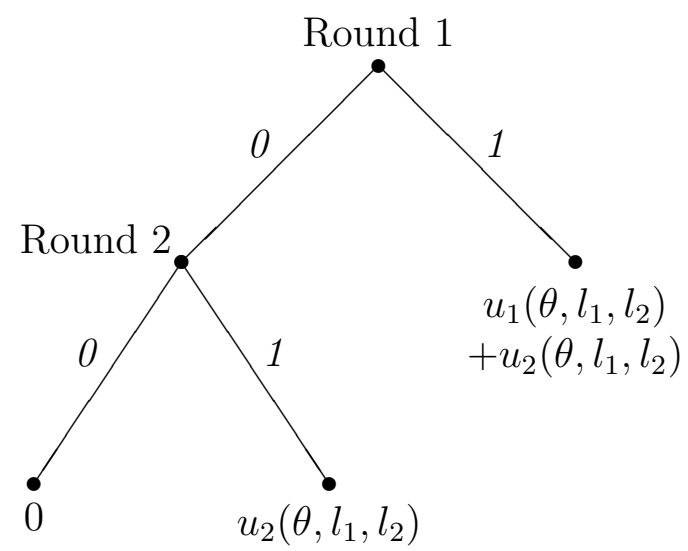

Figure 7: Variant of the dynamic game in which agents can delay investment.

Exit vs. delay option. We assumed that investment is reversible and that the safe action is irreversible. We have also examined a complementary variant of the dynamic game in which the investing is irreversible, whereas not investing is reversible - agents may delay investment, as in Figure 7. The two variants of the dynamic game can be mapped to each other by a careful relabeling of the actions, so we report only the analysis of the first variant. The studied effects, however, turn out to have opposite signs across the two games. To understand why, recall that the effects in the game with the exit option are driven by the technological constraint $l_{2} \leq l_{1}$. The opposite inequality $l_{2} \geq l_{1}$ holds in the variant with the delay option as agents who have not invested in round 1 can join the project in round 2. For this reason the first- and second-stage effects attain opposite signs. See Table 1 from the introduction for a summary and comparison.

Both actions reversible. Last, we have limited our analysis to setups with one reversible action. In the working version of this paper from 2008 we sketch an extension of the generalized Laplacian property to setups with many rounds and many options. Full generality of the Laplacian property and evaluation of the strategic consequences of reversibility options in general environments still remains an opportunity for future research. ${ }^{21}$

\footnotetext{
${ }^{21}$ One of the authors continues to examine the broad research topic of coordination and learning in a complementary paper of Mathevet and Steiner (2011). The two papers differ in the specification of the dynamic global game and in the techniques used in the analysis.
} 


\section{Conclusion}

Economically relevant coordination problems are rarely static. Typically, they are dynamic processes in which economic agents can postpone irreversible decisions in order to acquire additional information. We develop a modeling framework that incorporates learning and (ir)reversibility, while maintaining analytical tractability. The model allows for a qualitative assessment of the reversibility effects based solely on two features observable by an outside modeler. The first relevant feature is the (ir)reversibility of actions available to the economic agents. The second feature is the direction of the intertemporal payoff spillovers in between different stages of the coordination process. Based on these two features, the modeler or a policy maker can assess the effects of the reversibility option as summarized in Table 1 on page 4 .

The applicability of this dynamic framework can be demonstrated on the economic problem of creditor panic, discussed in the introduction. A large set of agents simultaneously decide whether to lend a fixed amount for a joint risky project. The agents can withdraw the investment in the interim stage of the project, or roll over the debt until its maturity. Such interaction corresponds to our model with reversible investment and an irreversible safe action, which conforms to the left column in Table 1. It is conceivable that the project exhibits forward payoff spillovers as high investment in the early stage may ceteris paribus increase profits in the late stage of the project due to inertia in the production process. On the contrary, backward spillovers are unlikely because the instantaneous profit from the first stage is presumably not causally influenced by the investment level in the later stage. Such a structure of payoff spillovers corresponds to the second row of Table 1. As the table specifies, the provision of an exit option enhances efficient coordination in this case. Although, as emphasized by Morris and Shin (2004), the exit option could lead to inefficient runs in the interim stage, this is, in this case, more than offset by the valuable flexibility provided by the option.

The conclusion is reversed if, preserving the structure of the payoff spillovers, agents have the option to delay investment instead of the exit option. ${ }^{22}$ As seen in the right column of Table 1, the delay option hampers efficient coordination in this case. These strategic consequences of the reversibility options could not be discussed in the original model of the creditor panic by Morris and Shin (2004). To fit the static global games framework, the authors kept the entry decisions exogenous, thus ignoring the intertemporal link between the first- and second-stage decisions on which this paper focuses.

In other cases, the structure of the payoff spillovers differs. In some applications of regime change games, the success of the attack and the payoffs for participation in the early and late wave of the attack depend only on the final size of the attack $l_{2}$ (as assumed in the main version of the model in Dasgupta 2007). In such cases the payoffs

\footnotetext{
${ }^{22}$ Heidhues and Melissas (2006), Section 3.2 falls into this category.
} 
exhibit backward but no forward spillovers, the delay option enhances and the exit option hampers efficient coordination.

\section{A Proofs}

We first introduce additional notation describing the error distributions. The supports of the marginal distributions of $\eta_{t}^{i}$ are, without loss of generality, ${ }^{23}$ assumed to be symmetric intervals $\left[-h_{t}, h_{t}\right]$ where $h_{t} \in(0,1]$. The joint density and the c.d.f. of the error pair $\left(\eta_{1}, \eta_{2}\right)$ are denoted $f$ and $F$, respectively. The marginal c.d.f. of $\eta_{t}^{i}$ is denoted $F_{t}$. In addition, $\eta_{\Delta}^{i}=\eta_{2}^{i}-\eta_{1}^{i}$ denotes the difference of the errors. The support of $\eta_{\Delta}^{i}$ is $\left[\underline{\eta}_{\Delta}, \bar{\eta}_{\Delta}\right]$ where $\underline{\eta}_{\Delta}=\min _{\left(\eta_{1}, \eta_{2}\right) \in H}\left(\eta_{2}-\eta_{1}\right)$ and $\bar{\eta}_{\Delta}=\max _{\left(\eta_{1}, \eta_{2}\right) \in H}\left(\eta_{2}-\eta_{1}\right)$. We denote the marginal c.d.f. of $\eta_{\Delta}^{i}$ by $F_{\Delta}$. See Figure 8 for illustration.

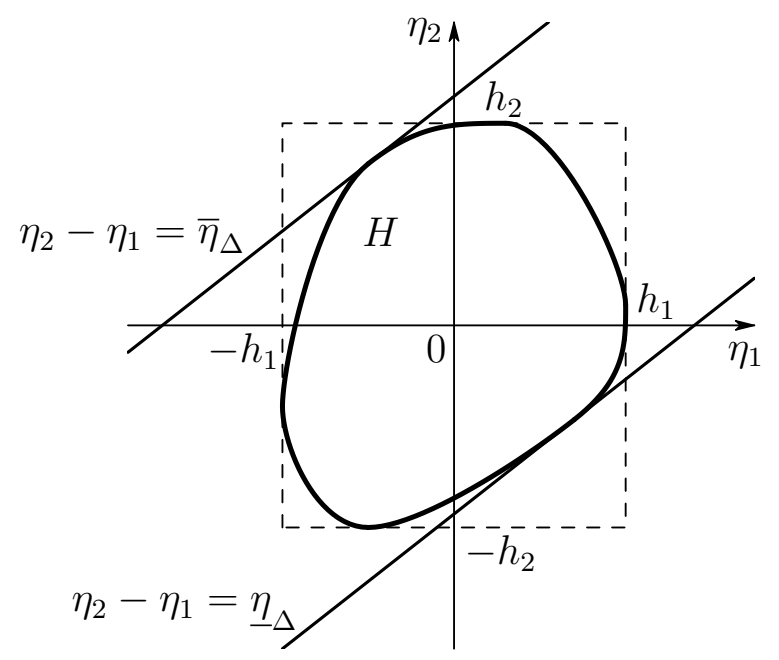

Figure 8: Notation describing error distributions.

\section{A.1 Proofs for Section 3}

Proof of Theorem 1 (Morris and Shin 2003). The theorem relies on three properties of the information structure. Errors $\eta_{1}^{i}$ are independent across agents, independent of $\theta$, and the agents hold uninformative prior belief about $\theta$.

The following computation verifies that the threshold type has the uniform strategic

\footnotetext{
${ }^{23}$ If the supports of the marginal distributions were not symmetric around 0 , agents would subtract an appropriate constant from their signals when forming posterior beliefs.
} 
belief; she assigns probability $z$ to the event that the aggregate action $l_{1}$ falls below $z$ :

$$
\begin{aligned}
& \operatorname{Pr}\left(l_{1}(\theta)<z \mid x_{1}^{i}=x_{1}^{*}\right)= \\
& \operatorname{Pr}\left(\operatorname{Pr}\left(x_{1}^{j}>x_{1}^{*} \mid \theta\right)<z \mid x_{1}^{i}=x_{1}^{*}\right)= \\
& \operatorname{Pr}\left(\operatorname{Pr}\left(\eta_{1}^{j}>\frac{x_{1}^{*}-\theta}{\sigma}\right)<z \mid x_{1}^{i}=x_{1}^{*}\right)= \\
& \operatorname{Pr}\left(1-F_{1}\left(\frac{x_{1}^{*}-\theta}{\sigma}\right)<z \mid x_{1}^{i}=x_{1}^{*}\right)= \\
& \operatorname{Pr}\left(1-F_{1}\left(\eta_{1}^{i}\right)<z\right)= \\
& \operatorname{Pr}\left(\eta_{1}^{i}>F_{1}^{-1}(1-z)\right)= \\
& 1-F_{1}\left(F_{1}^{-1}(1-z)\right)=z .
\end{aligned}
$$

Proof of Lemma 1. For convenience, let $\sigma=1$. Recall that we are proving

$$
E\left[s_{2}\left(\mathbf{x}^{i}\right) \cdot U_{2}\left(\mathbf{x}^{i}\right) \mid \partial L_{1}\right]=E\left[u_{2}\left(\theta, l_{1}, l_{2}\right) \mid \partial L_{2}\right]
$$

Let

$$
\begin{aligned}
& K_{s}=\left\{x_{2} \in\left[x_{1}^{*}+\underline{\eta}_{\Delta}, x_{1}^{*}+\bar{\eta}_{\Delta}\right]: s_{2}\left(x_{1}^{*}, x_{2}\right)=1\right\} \quad \text { and } \\
& K_{e}=\left\{x_{2} \in\left[x_{1}^{*}+\underline{\eta}_{\Delta}, x_{1}^{*}+\bar{\eta}_{\Delta}\right]: s_{2}\left(x_{1}^{*}, x_{2}\right)=0\right\}
\end{aligned}
$$

be the sets of the second-round signals $x_{2}$ such that type $\left(x_{1}^{*}, x_{2}\right)$ stays in, respectively exits the project.

We prove (10) by showing that both its left- and right-hand side are equal to

$$
\int_{K_{e}} 0 d F_{\Delta}\left(x_{2}-x_{1}^{*}\right)+\int_{K_{s}} U_{2}\left(x_{1}^{*}, x_{2}\right) d F_{\Delta}\left(x_{2}-x_{1}^{*}\right)
$$

We will use that $\eta_{\Delta}=x_{2}-x_{1}$ is independent of the events $\partial L_{1}$ and $\partial L_{2}$, and therefore the conditional distribution of $\eta_{\Delta} \mid \partial L_{t}$ equals the unconditional distribution $F_{\Delta}$. This independence will be demonstrated at the end of the proof.

The left-hand side of (10) equals (11) because $s_{2}\left(x_{1}^{*}, x_{2}\right)=0$ for $x_{2} \in K_{e}, s_{2}\left(x_{1}^{*}, x_{2}\right)=1$ for $x_{2} \in K_{s}$, and the c.d.f. of $x_{2} \mid \partial L_{1}$ is $F_{\Delta}\left(x_{2}-x_{1}^{*}\right)$.

We now focus on the right-hand side of (10). By the law of iterated expectations, the right-hand side of (10) is $E\left[E\left[u_{2}\left(\theta, l_{1}, l_{2}\right) \mid \mathbf{x}^{i}\right] \mid \partial L_{2}\right]=E\left[U_{2}\left(\mathbf{x}^{i}\right) \mid \partial L_{2}\right]$.

For each $x_{2}^{\prime} \in\left[x_{1}^{*}+\underline{\eta}_{\Delta}, x_{1}^{*}+\bar{\eta}_{\Delta}\right]$, define $\mathbf{x}\left(x_{2}^{\prime}\right)$ as the intersection of $\partial L_{2}$ with the line 
$x_{2}-x_{1}=x_{2}^{\prime}-x_{1}^{*}$. The intersection exists and is unique. ${ }^{24}$ We split the expectation $E\left[U_{2}(\mathbf{x}) \mid \partial L_{2}\right]$ into

$$
\int_{K_{e}} U_{2}\left(\mathbf{x}\left(x_{2}\right) d F_{\Delta}\left(x_{2}-x_{1}^{*}\right)+\int_{K_{s}} U_{2}\left(\mathbf{x}\left(x_{2}\right) d F_{\Delta}\left(x_{2}-x_{1}^{*}\right) .\right.\right.
$$

The first integral is identical to the first integral in (11) because if $x_{2} \in K_{e}$ then $\mathbf{x}\left(x_{2}\right)$ satisfies the indifference condition in round $2, U_{2}\left(\mathbf{x}\left(\eta_{\Delta}\right)\right)=0$. To see this, note that type $\left(x_{1}^{*}, x_{2}\right)$ exits and thus is not contained in $L_{2}$. Then, by the monotonicity property $\mathrm{P} 2$, $\mathbf{x}\left(x_{2}\right)$ is in the interior of $L_{1}$. Thus in any neighborhood of $\mathbf{x}\left(x_{2}\right)$ there exist $\mathbf{x}^{\prime}$ and $\mathbf{x}^{\prime \prime}$ such that $s_{2}\left(\mathbf{x}^{\prime}\right)=0$ and $s_{2}\left(\mathbf{x}^{\prime \prime}\right)=1$. Strategy $s_{2}(\mathbf{x})$ is optimal in round 2 (property P3) and hence $U_{2}\left(\mathbf{x}^{\prime}\right) \leq 0, U_{2}\left(\mathbf{x}^{\prime \prime}\right) \geq 0$. Then $U_{2}\left(\mathbf{x}\left(x_{2}\right)\right)=0$ from the continuity of expectations with respect to the signals. The second integral in (12) is identical to the second integral in (11) because if $x_{2} \in K_{s}$ then $\mathbf{x}\left(x_{2}\right)=\left(x_{1}^{*}, x_{2}\right)$ as the type $\left(x_{1}^{*}, x_{2}\right)$ lies on the boundary of $L_{2} \cdot{ }^{25}$

We now complete the proof by showing that $\eta_{1}^{i}, \eta_{2}^{i}$, and therefore $x_{2}-x_{1}=\eta_{2}^{i}-\eta_{1}^{i}$, are independent of the events $\partial L_{1}$ and $\partial L_{2}$. The argument applies not only to the boundaries of $L_{t}$ but to boundary of any diagonally monotone set $S$. Let $T_{d}(S)$ be a translation operator that translates a set $S \subseteq X$ in the direction of diagonal: $T_{d}(S)=$ $\{\mathbf{x} \in X: \mathbf{x}-(d, d) \in S\}$. We consider diagonal translations $T_{d}\left(\partial L_{t}\right)$. The uninformative prior and the independence of errors from $\theta$ imply that the conditional joint distribution of errors is invariant to diagonal translations: $\left(\eta_{1}^{i}, \eta_{2}^{i}\right)\left|\mathbf{x}=\left(\eta_{1}^{i}, \eta_{2}^{i}\right)\right|(\mathbf{x}+(d, d))$ for any $d \in \mathbb{R}$. Hence, the distribution of $\left(\eta_{1}^{i}, \eta_{2}^{i}\right) \mid\left(\mathbf{x}^{i} \in T_{d}\left(\partial L_{t}\right)\right)$ is identical for each $d$ and thus also equal to the unconditional distribution of $\left(\eta_{1}^{i}, \eta_{2}^{i}\right)$.

Proof of Lemma 2. For each type $\mathbf{x} \in X$, define $d(\mathbf{x})$ as such $d \in \mathbb{R}$ for which $\mathbf{x} \in T_{d}(\partial S)$ (see the proof of Lemma 1 for the definition of the translation operator $T_{d}$ ). We interpret $d(\mathbf{x})$ as a "distance" of $\mathbf{x}$ from $\partial S$ in the diagonal direction.

Let us consider virtual private signals $\tilde{x}^{i}=d\left(\mathbf{x}^{i}\right)$ and virtual errors $\tilde{\eta}^{i}=\tilde{x}^{i}-\theta$. The virtual information structure inherits all three properties that are sufficient for the static Laplacian property (Theorem 1). (i) $\theta$ is drawn from the uniform distribution (ii) The virtual errors $\tilde{\eta}^{i}$ are independent across agents, and (iii) independent of $\theta$. The properties (ii) and (iii) hold because, by the construction, the virtual error $\tilde{\eta}_{t}^{i}$ can be expressed as a function of $\left(\eta_{1}^{i}, \eta_{2}^{i}\right)$ and the original errors $\left(\eta_{1}^{i}, \eta_{2}^{i}\right)$ satisfy those properties.

Let $\tilde{x}^{*}=0$. From the definition of the virtual signals, event $\tilde{x}^{i}=\tilde{x}^{*}$ is identical to the event $\mathbf{x}^{i} \in \partial S$ and type $\mathbf{x}$ lies in (the closure of) $S$, if and only if $\tilde{x}^{i} \geq \tilde{x}^{*}$. See Figure 5

\footnotetext{
${ }^{24}$ The existence is assured by property P2: for sufficiently high $x_{1}$, type $\left(x_{1}, x_{1}+x_{2}^{\prime}-x_{1}^{*}\right) \in L_{2}$. For sufficiently low $x_{1}, x_{1}<x_{1}^{*}$ and then $\left(x_{1}, x_{1}+x_{2}^{\prime}-x_{1}^{*}\right) \notin L_{1} \supseteq L_{2}$. The uniqueness follows from the monotonicity property P1; each line parallel to the diagonal intersects $\partial L_{2}$ once.

${ }^{25}$ To see this, notice that $s_{2}\left(x_{1}^{*}, x_{2}\right)=1$ by the definition of $K_{s}$; therefore $\left(x_{1}^{*}, x_{2}\right) \in L_{2}$. On the other hand, $\left(x_{1}^{*}-\delta, x_{2}-\delta\right) \notin L_{1} \supseteq L_{2}$ for any $\delta>0$.
} 
for an illustration. Therefore, the conditional random variable $l_{S}(\theta) \mid \partial S$ is identical to

$$
\operatorname{Pr}\left(\tilde{x}^{j} \geq \tilde{x}^{*} \mid \theta\right) \mid\left(\tilde{x}^{i}=\tilde{x}^{*}\right)
$$

and the last conditional random variable is uniformly distributed on $[0,1]$ by the static Laplacian property, Theorem 1.

\section{A.2 Proofs for Section 4}

The continuation game $\Gamma_{2}\left(x_{1}^{*}\right)$ is a static Bayesian game in which agents $i \in[0,1]$ observe their types $\mathbf{x}^{i} \in X$ and simultaneously choose strategy $s_{2}: X \longrightarrow\{0,1\}$. The joint distribution of $\left(\theta, x_{1}, x_{2}\right)$ is as in the dynamic game. The payoff of type $\mathbf{x}$ choosing action $s_{2}(\mathbf{x})$ is $s_{2}(\mathbf{x}) \cdot u_{2}\left(\theta, l_{1}(\theta), l_{2}(\theta)\right)$ where $l_{1}(\theta), l_{2}(\theta)$ are, as before, the first and the second stage investment levels defined in the dynamic game, when all agents follow threshold $x_{1}^{*}$ in round 1 and strategy profile $\left(s_{2}^{i}\right)_{i}$ in round 2 .

We define rationalizable strategies in the continuation game as follows. Strategy profile in $\Gamma_{2}\left(x_{1}^{*}\right)$ is a mapping $\Sigma_{2}(i)$ specifying strategy $s_{2}^{i}$ for each agent $i$. The best response set $B R_{2}\left(\mathbf{x}, \Sigma_{2}\right) \subseteq\{0,1\}$ of type $\mathbf{x}$ against profile $\Sigma_{2}$ contains action 1 if $E\left[u_{2}\left(\theta, l_{1}, l_{2}\right) \mid \mathbf{x}\right] \geq 0$ and action 0 if $E\left[u_{2}\left(\theta, l_{1}, l_{2}\right) \mid \mathbf{x}\right] \leq 0$ under $\Sigma_{2}$. We let $S_{2}^{0}$ denote the set of all strategies, and define $S_{2}^{k}$ recursively for $k=1,2, \ldots$ : strategy $s_{2} \in S_{2}^{k}$ if and only if (i) $s_{2} \in S_{2}^{k-1}$, and (ii) for each type $\mathbf{x} \in X$ there exists profile $\Sigma_{2}$ such that $\Sigma_{2}(i) \in S_{2}^{k-1}$ for all agents $i$ and $s_{2}(\mathbf{x}) \in B R_{2}\left(\mathbf{x}, \Sigma_{2}\right)$. The set of rationalizable strategies is $S_{2}^{*}=\bigcap_{k} S_{2}^{k}$.

Let $s_{1}$ be the threshold strategy in round 1 with threshold $x_{1}^{*}$. The following lemma is an auxiliary result used in the proof of Lemma 3.

Lemma 5. For each $x_{1}^{*} \in \mathbb{R}$, the continuation game $\Gamma_{2}\left(x_{1}^{*}\right)$ has a unique rationalizable strategy $s_{2}$. Pair $\left(s_{1}, s_{2}\right)$ satisfies properties P1, P2, and P3 from Section 3.

Proof of Lemma 5. For convenience, we let $\sigma=1$.

We first construct the largest and smallest rationalizable strategies using a contagion argument and then show that they coincide by an adaptation of the translation argument from Frankel, Morris and Pauzner (2003).

In the first step we analyze the maximal and the minimal rationalizable strategy in $\Gamma_{2}\left(x_{1}^{*}\right)$. See van Zandt and Vives (2007) for general characterization of extreme rationalizable strategies in Bayesian games with strategic complementarities. Our problem differs in certain details of the setting, such as continuous vs. discrete set of agents, and so we give a direct argument.

For a symmetric strategy profile $\left(s_{1}, s_{2}\right)$, let $L_{1}=\left\{\mathbf{x} \in X: s_{1}\left(x_{1}\right)=1\right\}, I_{2}=\{\mathbf{x} \in$ $\left.X: s_{2}(\mathbf{x})=1\right\}$, and define $\tilde{U}_{2}\left(\mathbf{x}, L_{1}, I_{2}\right)=E\left[u_{2}\left(\theta, l_{1}, l_{2}\right) \mid \mathbf{x}\right]$ to be the expected second stage payoff under the symmetric profile $\left(s_{1}, s_{2}\right)$. 
Let $\underline{I}_{2}^{(0)}=\emptyset$, and $\bar{I}_{2}^{(0)}=X$, and for $k=0,1 \ldots$ define recursively

$$
\begin{aligned}
& \underline{I}_{2}^{(k+1)}=\left\{\mathbf{x} \in X: \tilde{U}_{2}\left(\mathbf{x}, L_{1}, \underline{I}_{2}^{(k)}\right)>0\right\}, \\
& \bar{I}_{2}^{(k+1)}=\left\{\mathbf{x} \in X: \tilde{U}_{2}\left(\mathbf{x}, L_{1}, \bar{I}_{2}^{(k)}\right) \geq 0\right\} .
\end{aligned}
$$

The interpretation of these sets is as follows. Action 1 is unique rationalizable action in $\Gamma_{2}\left(x_{1}^{*}\right)$ on types in $\underline{I}_{2}^{(k)}$ and 0 is unique rationalizable action in $\Gamma_{2}\left(x_{1}^{*}\right)$ on types in $X \backslash \bar{I}_{2}^{(k)}$ after $k$ iterations.

By induction, all following statements hold: The sets $\underline{I}_{2}^{(k)}, \bar{I}_{2}^{(k)}$ are diagonally monotone sets and $\underline{I}_{2}^{(k)} \subseteq \bar{I}_{2}^{(k)}, \bar{I}_{2}^{(k+1)} \subseteq \bar{I}_{2}^{(k)}$, and $\underline{I}_{2}^{(k+1)} \supseteq \underline{I}_{2}^{(k)}$ for all $k$. Next, define sets $\underline{I}_{2}=\bigcup_{k} \underline{I}_{2}^{(k)}$ and $\bar{I}_{2}=\bigcap_{k} \bar{I}_{2}^{(k)}$. Action 1 is the unique rationalizable action on $\underline{I}_{2}$ and 0 is the unique rationalizable action on $X \backslash \bar{I}_{2}$. From the properties of the sets $\underline{I}_{2}^{(k)}, \bar{I}_{2}^{(k)}$, the sets $\underline{I}_{2}, \bar{I}_{2}$ are diagonally monotone sets and $\bar{I}_{2} \supseteq \underline{I}_{2}$.

The second step of the proof adapts the translation argument of Frankel, Morris, and Pauzner (2003). We prove that the sets $\underline{I}_{2}$ and $\bar{I}_{2}$ have identical boundaries, $\partial \bar{I}_{2}=\partial \underline{I}_{2}$. Suppose for contradiction that $\partial \bar{I}_{2} \neq \partial \underline{I}_{2}$.

Let us first recall notation introduced at the beginning of the Appendix: $\eta_{\Delta}^{i} \in\left[\underline{\eta}_{\Delta}, \bar{\eta}_{\Delta}\right]$ denotes errors' difference $\eta_{2}^{i}-\eta_{1}^{i}$, and with $\sigma=1$ we also have $\eta_{\Delta}^{i}=x_{2}^{i}-x_{1}^{i}$. In addition, let functions $\underline{\mathbf{x}}:\left[\underline{\eta}_{\Delta}, \bar{\eta}_{\Delta}\right] \longrightarrow \partial \underline{I}_{2}$ and $\overline{\mathbf{x}}:\left[\underline{\eta}_{\Delta}, \bar{\eta}_{\Delta}\right] \longrightarrow \partial \bar{I}_{2}$ denote the intersections of the line $x_{2}-x_{1}=\eta_{\Delta}$ with $\partial \underline{I}_{2}$ and $\partial \bar{I}_{2}$, respectively.

By Milgrom and Roberts (1990), the largest and the smallest rationalizable strategy each constitutes a symmetric equilibrium in $\Gamma_{2}\left(x_{1}^{*}\right)$. Hence, the types $\underline{\mathbf{x}}\left(\eta_{\Delta}\right)$ and $\overline{\mathbf{x}}\left(\eta_{\Delta}\right)$ satisfy the indifference conditions, $\tilde{U}_{2}\left(\underline{\mathbf{x}}\left(\eta_{\Delta}\right), L_{1}, \underline{I}_{2}\right)=\tilde{U}_{2}\left(\overline{\mathbf{x}}\left(\eta_{\Delta}\right), L_{1}, \bar{I}_{2}\right)=0$. Also, $\underline{\mathbf{x}}\left(\eta_{\Delta}\right) \geq \overline{\mathbf{x}}\left(\eta_{\Delta}\right)$ for all $\eta_{\Delta}$ because the sets $\underline{I}_{2}, \bar{I}_{2}$ are diagonally monotone sets and $\underline{I}_{2} \subseteq \bar{I}_{2}$.

As the last property, note that the functions $\underline{\mathbf{x}}\left(\eta_{\Delta}\right), \overline{\mathbf{x}}\left(\eta_{\Delta}\right)$ are continuous. Consider $\underline{\mathbf{x}}\left(\eta_{\Delta}\right)$ at $\eta_{0}$, denote $\mathbf{x}_{0}=\underline{\mathbf{x}}\left(\eta_{0}\right),{ }^{26}$ and let

$$
\underline{U}(\mathbf{x})=\tilde{U}_{2}\left(\mathbf{x}, L_{1}, \underline{I}_{2}\right) .
$$

Then $\underline{U}\left(\mathbf{x}_{0}-(\varepsilon / 2, \varepsilon / 2)\right)<0<\underline{U}\left(\mathbf{x}_{0}+(\varepsilon / 2, \varepsilon / 2)\right)$ by assumptions A1 and A2. Using the continuity of payoff expectations with respect to signals, there exists $\bar{\delta} \in(0, \varepsilon / 2)$ such that for all $\delta \in(-\bar{\delta}, \bar{\delta})$ the following inequalities hold:

$$
\underline{U}\left(\mathbf{x}_{0}-(\varepsilon / 2, \varepsilon / 2)+(-\delta, \delta)\right)<0<\underline{U}\left(\mathbf{x}_{0}+(\varepsilon / 2, \varepsilon / 2)+(-\delta, \delta)\right) .
$$

Moreover, both $\mathbf{x}_{0} \pm(\varepsilon / 2, \varepsilon / 2)+(-\delta, \delta) \in B$, where $B$ is a ball with radius $\varepsilon$ around $\mathbf{x}_{0}$.. Consider now $\eta$ such that $\left|\eta-\eta_{0}\right|<\bar{\delta}$ and let $\delta=\eta-\eta_{0}$. Then it follows from

\footnotetext{
${ }^{26}$ The proof for $\overline{\mathbf{x}}\left(\eta_{\Delta}\right)$ is similar.
} 
(13) and monotonicity with respect to $\varepsilon$ that $\underline{\mathbf{x}}(\eta)=\underline{U}\left(\mathbf{x}_{0}+\left(\varepsilon^{\prime}, \varepsilon^{\prime}\right)+(-\delta, \delta)\right)$ for some $\varepsilon^{\prime} \in(-\varepsilon / 2, \varepsilon / 2)$. This, in turn, implies that $\underline{\mathbf{x}}(\eta) \in B$, which completes the proof of the continuity.

Next, we define function $\zeta:\left[\underline{\eta}_{\Delta}, \bar{\eta}_{\Delta}\right] \longrightarrow[0,+\infty)$ as $\zeta\left(\eta_{\Delta}\right)=\underline{x}_{1}\left(\eta_{\Delta}\right)-\bar{x}_{1}\left(\eta_{\Delta}\right)$ where $\underline{x}_{1}, \bar{x}_{1}$ are the first coordinates of $\underline{\mathbf{x}}, \overline{\mathbf{x}}$. Notice that $\zeta\left(\eta_{\Delta}\right) \geq 0$ for all $\eta_{\Delta}$ because $\underline{x}_{1}\left(\eta_{\Delta}\right) \geq$ $\bar{x}_{1}\left(\eta_{\Delta}\right)$. The function $\zeta$ is continuous and hence it attains a maximum on the compact set $\left[\underline{\eta}_{\Delta}, \bar{\eta}_{\Delta}\right]$ at some value $\hat{\eta}_{\Delta}$. The maximal value $\zeta\left(\hat{\eta}_{\Delta}\right)$ is strictly positive if and only if the boundaries $\partial \underline{I}_{2}$ and $\partial \bar{I}_{2}$ differ.

Similarly to $\underline{U}(\mathbf{x})$, let

$$
\begin{aligned}
\bar{U}(\mathbf{x}) & =\tilde{U}_{2}\left(\mathbf{x}, L_{1}, \bar{I}_{2}\right), \\
\bar{U}^{\prime}(\mathbf{x}) & =\tilde{U}_{2}\left(\mathbf{x}, L_{1}, T_{\zeta\left(\hat{\eta}_{\Delta}\right)}\left(\bar{I}_{2}\right)\right),
\end{aligned}
$$

where $T$ is the translation operator along the diagonal, as defined in the proof of Lemma 1 $\left(T_{d}(S)\right.$ is translation of the set $S$ by vector $\left.(d, d)\right)$.

We now establish two inequalities. By construction, $T_{\zeta\left(\hat{\eta}_{\Delta}\right)}\left(\bar{I}_{2}\right)$ is a subset of the closure of $\underline{I}_{2}$ and therefore by action monotonicity

$$
\bar{U}^{\prime}(\mathbf{x}) \leq \underline{U}(\mathbf{x})
$$

for all $\mathbf{x} \in X$. Also,

$$
\bar{U}^{\prime}(\mathbf{x})>\bar{U}\left(\mathbf{x}-\zeta\left(\hat{\eta}_{\Delta}\right) \cdot(1,1)\right)
$$

because type $\mathbf{x}$ under $I_{2}=T_{\zeta\left(\hat{\eta}_{\Delta}\right)}\left(\bar{I}_{2}\right)$ has identical belief about the aggregate action in round 2 as type $\mathbf{x}-\zeta\left(\hat{\eta}_{\Delta}\right) \cdot(1,1)$ under $I_{2}=\bar{I}_{2}$, but the belief of the latter about $\left(\theta, l_{1}\right)$ is stochastically dominated (in the sense of the first order stochastic dominance) by the belief about $\left(\theta, l_{1}\right)$ of the former type; strict inequality holds because $u_{2}$ increases in $\theta$.

Finally, consider the type $\underline{\mathbf{x}}\left(\hat{\eta}_{\Delta}\right)=\overline{\mathbf{x}}\left(\hat{\eta}_{\Delta}\right)+\zeta\left(\hat{\eta}_{\Delta}\right) \cdot(1,1)$. The types $\underline{\mathbf{x}}\left(\hat{\eta}_{\Delta}\right)$, and $\overline{\mathbf{x}}\left(\hat{\eta}_{\Delta}\right)$ satisfy the indifference conditions, and hence $\underline{U}\left(\underline{\mathbf{x}}\left(\hat{\eta}_{\Delta}\right)\right)=0, \bar{U}\left(\overline{\mathbf{x}}\left(\hat{\eta}_{\Delta}\right)\right)=0$. On one hand, using inequality $(14), \bar{U}^{\prime}\left(\underline{\mathbf{x}}\left(\hat{\eta}_{\Delta}\right)\right) \leq \underline{U}\left(\underline{\mathbf{x}}\left(\hat{\eta}_{\Delta}\right)\right)=0$, but on the other hand, by inequality (15), $\bar{U}^{\prime}\left(\underline{\mathbf{x}}\left(\hat{\eta}_{\Delta}\right)\right)>\bar{U}\left(\overline{\mathbf{x}}\left(\hat{\eta}_{\Delta}\right)\right)=0$, which establishes the contradiction.

Additionally, notice that the unique rationalizable strategy $s_{2}$ in $\Gamma_{2}\left(x_{1}^{*}\right)$ attains 1 for sufficiently high types; the existence of the upper dominance region assures that action 1 is dominant for sufficiently high types in $\Gamma_{2}\left(x_{1}^{*}\right)$.

Proof of Lemma 3. We again let $\sigma=1$ in this proof. We obtain $m_{d y n}\left(x_{1}^{*}\right)>0$ for sufficiently high $x_{1}^{*}$ and $m_{d y n}\left(x_{1}^{*}\right)<0$ for sufficiently low $x_{1}^{*}$ by the existence of the dominance regions. 
In the rest of the proof we establish the monotonicity of $m_{d y n}\left(x_{1}^{*}\right)$. Consider $L_{1}\left(x_{1}^{*}\right)$ and the sets $\underline{I}_{2}^{(k)}\left(x_{1}^{*}\right)$ as defined for the continuation game $\Gamma_{2}\left(x_{1}^{*}\right)$ in the proof of Lemma $5 .^{27}$ Assume $x_{1}^{\prime}>x_{1}^{*}$ and let $d=x_{1}^{\prime}-x_{1}^{*}$. We prove by induction that $\underline{I}_{2}\left(x_{1}^{\prime}\right) \supseteq T_{d}\left(\underline{I}_{2}\left(x_{1}^{*}\right)\right)$.

First, notice that, trivially,

$$
\underline{I}_{2}^{(0)}\left(x_{1}^{\prime}\right) \supseteq T_{d}\left(\underline{I}_{2}^{(0)}\left(x_{1}^{*}\right)\right)
$$

because $I_{2}^{(0)}\left(x_{1}^{*}\right)=I_{2}^{(0)}\left(x_{1}^{\prime}\right)=\emptyset$. For the induction step, assume $\underline{I}_{2}^{(k)}\left(x_{1}^{\prime}\right) \supseteq T_{d}\left(\underline{I}_{2}^{(k)}\left(x_{1}^{*}\right)\right)$. First,

$$
\tilde{U}_{2}\left(\mathbf{x}, L_{1}\left(x_{1}^{*}\right), \underline{I}_{2}^{(k)}\left(x_{1}^{*}\right)\right)<\tilde{U}_{2}\left(\mathbf{x}+(d, d), T_{d}\left(L_{1}\left(x_{1}^{*}\right)\right), T_{d}\left(\underline{I}_{2}^{(k)}\left(x_{1}^{*}\right)\right)\right)
$$

by the strict state monotonicity A1. Second, by the action monotonicity A2 and by the induction assumption, the right hand side is smaller or equal than

$$
\tilde{U}_{2}\left(\mathbf{x}+(d, d), T_{d}\left(L_{1}\left(x_{1}^{*}\right)\right), \underline{I}_{2}^{(k)}\left(x_{1}^{\prime}\right)\right)=\tilde{U}_{2}\left(\mathbf{x}+(d, d), L_{1}\left(x_{1}^{\prime}\right), \underline{I}_{2}^{(k)}\left(x_{1}^{\prime}\right)\right) .
$$

Summarizing

$$
\tilde{U}_{2}\left(\mathbf{x}, L_{1}\left(x_{1}^{*}\right), \underline{I}_{2}^{(k)}\left(x_{1}^{*}\right)\right)<\tilde{U}_{2}\left(\mathbf{x}+(d, d), L_{1}\left(x_{1}^{\prime}\right), \underline{I}_{2}^{(k)}\left(x_{1}^{\prime}\right)\right)
$$

and, using definition of $\underline{I}_{2}^{(k+1)}$, we closed the induction step; $\underline{I}_{2}^{(k+1)}\left(x_{1}^{\prime}\right) \supseteq T_{d}\left(\underline{I}_{2}^{(k+1)}\left(x_{1}^{*}\right)\right)$.

We have established $\underline{I}_{2}\left(x_{1}^{\prime}\right) \supseteq T_{x_{1}^{\prime}-x_{1}^{*}}\left(\underline{I}_{2}\left(x_{1}^{*}\right)\right)$. Then, $D_{t}\left(x_{1}^{\prime}\right)>D_{t}\left(x_{1}^{*}\right)$ for $t=1,2$ because round 1 belief at signal $x_{1}^{\prime}$ about $\left(\theta, l_{1}, l_{2}\right)$ under strategy profile induced by the sets $L_{1}\left(x_{1}^{\prime}\right), I_{2}\left(x_{1}^{\prime}\right)$ dominate round 1 belief at signal $x_{1}^{*}$ about $\left(\theta, l_{1}, l_{2}\right)$ under $L_{1}\left(x_{1}^{*}\right)$, $I_{2}\left(x_{1}^{*}\right)$. The strict inequality follows from the strict state monotonicity A1. Therefore, $m_{d y n}\left(x_{1}^{*}\right)=D_{1}\left(x_{1}^{*}\right)+D_{2}\left(x_{1}^{*}\right)$ is increasing in $x_{1}^{*}$.

Proof of Proposition 1. The proof follows from Lemmas 3 and 5.

\section{A.3 Auxiliary Results for Section 5}

Before proving Propositions 2 and 3 we formulate and prove Lemmas 6, 7, 8 and 9 on the limiting behavior of the static and dynamic games when $\sigma \rightarrow 0$.

Lemma 6. The limit $m_{d y n}^{*}\left(x_{1}^{*}\right)=\lim _{\sigma \rightarrow 0} m_{d y n}\left(x_{1}^{*} ; \sigma\right)$ exists, is continuous and increasing in $x_{1}^{*}$.

Proof of Lemma 6. First, we introduce additional notation. Let $\tilde{F}\left(z_{1}, z_{2}\right)=\operatorname{Pr}\left(\eta_{1}^{i} \geq\right.$ $z_{1}$ and $\left.\eta_{2}^{i} \geq z_{2}\right)$ denote the complementary cumulative distribution function of $\left(\eta_{1}^{i}, \eta_{2}^{i}\right)$.

\footnotetext{
${ }^{27}$ We highlight the dependence on the round 1 threshold by the additional argument.
} 
As $\eta_{2}^{i}$ and $\eta_{\Delta}^{i}$ are independent, the set $H$ is a parallelogram and $\bar{\eta}_{\Delta}=-\underline{\eta}_{\Delta}=h_{1}-h_{2}$. When $x_{2}^{i}$ is sufficient statistic, agent $i$ 's decision in round 2 depends only on $x_{2}^{i}$ and not on $x_{1}^{i}$. Thus, $s_{2}\left(x_{2}^{i}\right)$ is a monotone function $\mathbb{R} \longrightarrow\{0,1\}$ with a threshold denoted by $x_{2}^{*}$. We denote $\eta^{*}=\frac{x_{2}^{*}-x_{1}^{*}}{\sigma}$.

For the purpose of this proof we need more precise but more elaborate notation describing mappings between $\theta, l_{1}$ and $l_{2}$. Let $\vartheta_{t}\left(l_{t} ; x_{1}^{*}, \sigma\right)$ be the inverse function of $l_{t}\left(\theta ; x_{1}^{*}, \sigma\right)$ with respect to $\theta$. Omitting the dependence on $x_{1}^{*}$ and $\sigma$, let $\lambda_{1}\left(l_{2}\right)=l_{1}\left(\vartheta_{2}\left(l_{2}\right)\right)$ denote investment level $l_{1}$ as a function of $l_{2}$. Similarly, $\lambda_{2}\left(l_{1}\right)=l_{2}\left(\vartheta_{1}\left(l_{1}\right)\right)$.

We divide the proof into three cases depending on the value of $x_{1}^{*}$.

Case (a). If $u_{2}\left(x_{1}^{*}, 0,0\right)>0$, then $u_{2}\left(x_{1}^{*}-\sigma h_{1}, 0,0\right)>0$ for sufficiently small $\sigma$. Then, all types with $x_{1}^{i} \geq x_{1}^{*}$ strictly prefer to stay in the project in the continuation game $\Gamma_{2}\left(x_{1}^{*} ; \sigma\right)$. Thus, $l_{1}\left(\theta ; x_{1}^{*}, \sigma\right)=l_{2}\left(\theta ; x_{1}^{*}, \sigma\right)$ and so $m_{d y n}\left(x_{1}^{*} ; \sigma\right)=m_{s t}\left(x_{1}^{*} ; \sigma\right)$ and $\lim _{\sigma \rightarrow 0} m_{d y n}\left(x_{1}^{*} ; \sigma\right)=m_{s t}^{*}\left(x_{1}^{*}\right)=\int_{0}^{1}\left(u_{1}\left(x_{1}^{*}, l, l\right)+u_{2}\left(x_{1}^{*}, l, l\right)\right) d l$.

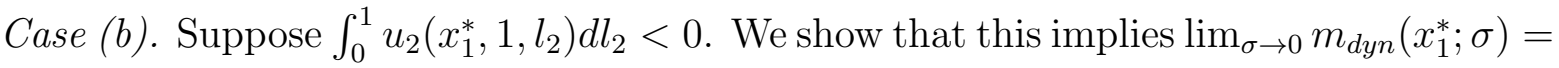
$\int_{0}^{1} u_{1}\left(x_{1}^{*}, l_{1}, 0\right) d l_{1}$. We first establish that $x_{2}^{*}(\sigma) \geq x_{1}^{*}+\sigma\left(h_{1}+h_{2}\right)$ when $\sigma$ is sufficiently small. Suppose the opposite inequality, $x_{2}^{*}(\sigma)<x_{1}^{*}+\sigma\left(h_{1}+h_{2}\right)$. Then, for type $\mathbf{x}$ who observes signal $x_{2}^{*}$ in round 2 we obtain

$$
U_{2}(\mathbf{x})<\int_{0}^{1} u_{2}\left(x_{1}^{*}+\sigma\left(h_{1}+2 h_{2}\right), 1, l_{2}\right) d l_{2},
$$

The above inequality is based on three observations. First, an agent with signal $x_{2}^{*}$ in round 2 knows that the fundamental does not exceed the value $x_{2}^{*}+\sigma h_{2}<x_{1}^{*}+\sigma\left(h_{1}+\right.$ $\left.h_{2}\right)+\sigma h_{2}=x_{1}^{*}+\sigma\left(h_{1}+2 h_{2}\right)$. Second, the investment level $l_{1}$ can be at most 1 . Third, the second round belief about $l_{2}$ of the agent who observes $x_{2}^{*}$ is stochastically dominated by the uniform distribution on $[0,1]$, as only fellow agents who observe second round signal $x_{2}^{i} \geq x_{2}^{*}$ can possibly participate in the second stage. The right-hand side of (16) is negative for sufficiently small $\sigma$, which contradicts the indifference condition that the type $x_{2}^{*}$ in round 2 must satisfy. Thus, indeed $x_{2}^{*}(\sigma) \geq x_{1}^{*}+\sigma\left(h_{1}+h_{2}\right)$ for small $\sigma$.

The last inequality implies that the types with $x_{1}^{i}=x_{1}^{*}$ do not reach action path 11 in $\Gamma_{2}\left(x_{1}^{*} ; \sigma\right)$, and hence $D_{2}\left(x_{1}^{*} ; \sigma\right)=0$. Moreover, $\vartheta_{1}\left(l_{1} ; x_{1}^{*}, \sigma\right) \leq x_{1}^{*}+\sigma h_{1}$ for all $l_{1} \in(0,1)$. Therefore, $\lambda_{2}\left(l_{1}\right) \leq l_{2}\left(x_{1}^{*}+\sigma h_{1}\right)$. But $l_{2}\left(x_{1}^{*}+\sigma h_{1}\right)=0$ because we established that agents with the second signal at most $x_{1}^{*}+\sigma\left(h_{1}+h_{2}\right)$ do not invest in the continuation game $\Gamma_{2}\left(x_{1}^{*} ; \sigma\right)$. Thus, $\lambda_{2}\left(l_{1}\right)=0$ for all $l_{1} \in(0,1)$ and for sufficiently small $\sigma$.

Case (c). Consider the case when $u_{2}\left(x_{1}^{*}, 0,0\right) \leq 0 \leq \int_{0}^{1} u_{2}\left(x_{1}^{*}, 1, l_{2}\right) d l_{2}$. This case requires an additional notation. Let $a_{1}=\frac{x_{1}^{*}-\theta}{\sigma}, a_{2}=\frac{x_{2}^{*}-\theta}{\sigma}=a_{1}+\eta^{*}$. Note that $l_{1}\left(x_{1}^{*}-\right.$ $\left.\sigma a_{1} ; x_{1}^{*}, \sigma\right)$ depends only on $a_{1}$ and is independent of $x_{1}^{*}, x_{2}^{*}$ and $\sigma$. Similarly $l_{2}\left(x_{1}^{*}-\right.$ $\left.\sigma a_{1} ; x_{1}^{*}, \sigma\right)$ depends only on $a_{1}$ and $\eta^{*}$. To see this, recall that $l_{1}\left(\theta ; x_{1}^{*}, \sigma\right)=\operatorname{Pr}\left(x_{1}^{i} \geq\right.$ $\left.x_{1}^{*} \mid \theta\right)=\operatorname{Pr}\left(\eta_{1}^{i} \geq \frac{x_{1}^{*}-\theta}{\sigma} \mid \theta\right)$, and thus, $l_{1}\left(x_{1}^{*}-\sigma a_{1} ; x_{1}^{*}, \sigma\right)=\operatorname{Pr}\left(\eta_{1}^{i} \geq a_{1}\right)=\tilde{F}\left(a_{1},-h_{2}\right)$. 
Similarly, $l_{2}\left(x_{1}^{*}-\sigma a_{1} ; x_{1}^{*}, \sigma\right)=\operatorname{Pr}\left(\eta_{1}^{i} \geq a_{1}\right.$ and $\left.\eta_{2}^{i} \geq a_{1}+\eta^{*}\right)=\tilde{F}\left(a_{1}, a_{1}+\eta^{*}\right)$.

Agent receiving the threshold signal $x_{2}^{*}$ in round 2 is indifferent between actions 0 and 1. In the above notation, and after transformation $\theta=x_{1}^{*}-\sigma\left(a_{2}-\eta^{*}\right)$, the indifference condition can be written as

$$
J(\eta ; \sigma)=\int_{-h_{2}}^{h_{2}} u_{2}\left(x_{1}^{*}-\sigma\left(a_{2}-\eta\right), \tilde{F}\left(a_{2}-\eta,-h_{2}\right), \tilde{F}\left(a_{2}-\eta, a_{2}\right)\right) f_{2}\left(a_{2}\right) d a_{2}=0 .
$$

Observe that for $\sigma>0$, function $J(\eta ; \sigma)$ is increasing and continuous in $\eta$ and due to the existence of dominance regions, it attains both positive and negative values. Thus, for every $\sigma>0$ there exists unique $\eta=\eta^{*}(\sigma)$ such that $J(\eta ; \sigma)=0$. For $\sigma=0$ : $J(\eta ; 0)$ is increasing in $\eta$ as well, by the strict monotonicity of $u_{2}$ in $l_{2}$. Therefore, the equation $J(\eta ; 0)=0$ has at most one solution.

Now, for $\eta=-\left(h_{1}+h_{2}\right)$ and for all $a_{2} \geq-h_{2}$, we have $\tilde{F}\left(a_{2}-\eta,-h_{2}\right)=\tilde{F}\left(a_{2}-\right.$ $\left.\eta, a_{2}\right)=0$. Moreover, for $\eta=h_{1}+h_{2}$ and $a_{2} \leq h_{2}$, we have $\tilde{F}\left(a_{2}-\eta,-h_{2}\right)=1$ and $\tilde{F}\left(a_{2}-\eta, a_{2}\right)=\operatorname{Pr}\left(\eta_{2}^{i} \geq a_{2}\right)=1-F_{2}\left(a_{2}\right)$, where $F_{2}$ is the c.d.f. of $\eta_{2}^{i}$. Summing up,

$$
J\left(-\left(h_{1}+h_{2}\right) ; 0\right)=u_{2}\left(x_{1}^{*}, 0,0\right) \leq 0 \leq \int_{0}^{1} u_{2}\left(x_{1}^{*}, 1, l_{2}\right) d l_{2}=J\left(h_{1}+h_{2} ; 0\right) .
$$

Therefore, the equation $J(\eta ; 0)=0$ has indeed a unique solution and that solution lies in the interval $\left[-\left(h_{1}+h_{2}\right), h_{1}+h_{2}\right]$; denote it $\eta^{* *}$. It follows that $\lim _{\sigma \rightarrow 0} \eta^{*}(\sigma)=\eta^{* *}$ and $\eta^{* *}$ is continuous and decreasing in $x_{1}^{*}$, for $x_{1}^{*}$ such that $u_{2}\left(x_{1}^{*}, 0,0\right) \leq 0 \leq \int_{0}^{1} u_{2}\left(x_{1}^{*}, 1, l_{2}\right) d l_{2}$.

Let us now study $\lim _{\sigma \rightarrow 0} D_{t}\left(x_{1}^{*} ; \sigma\right)$, for the range of $x_{1}^{*}$ considered in (c). In order to study $\lim _{\sigma \rightarrow 0} D_{1}\left(x_{1}^{*} ; \sigma\right)$, let us first denote $\tilde{F}_{1}^{-1}(l)$ the inverse function to $\tilde{F}\left(z,-h_{2}\right)$ with respect to $z$. Then, $\vartheta_{1}\left(l_{1}\right)=x_{1}^{*}-\sigma \tilde{F}_{1}^{-1}\left(l_{1}\right)$ and $\lambda_{2}\left(l_{1}\right)=\tilde{F}\left(\tilde{F}_{1}^{-1}\left(l_{1}\right), \tilde{F}_{1}^{-1}\left(l_{1}\right)+\eta^{*}(\sigma)\right)$. Both are continuous in $x_{1}^{*}$ and $\sigma$, and in the limit $\sigma \rightarrow 0$, we have $\vartheta_{1}\left(l_{1}\right) \rightarrow x_{1}^{*}$ and $\lambda_{2}\left(l_{1}\right) \rightarrow \lambda_{2}^{*}\left(l_{1} ; x_{1}^{*}\right)=\tilde{F}\left(\tilde{F}_{1}^{-1}\left(l_{1}\right), \tilde{F}_{1}^{-1}\left(l_{1}\right)+\eta^{* *}\left(x_{1}^{*}\right)\right)$. Note that the latter is non-increasing in $\eta^{* *}$. Thus, the limit

$$
\lim _{\sigma \rightarrow 0} D_{1}\left(x_{1}^{*} ; \sigma\right)=\lim _{\sigma \rightarrow 0} \int_{0}^{1} u_{1}\left(x_{1}^{*}-\sigma \tilde{F}_{1}^{-1}\left(l_{1}\right), l_{1}, \lambda_{2}\left(l_{1}\right)\right) d l_{1}=\int_{0}^{1} u_{1}\left(x_{1}^{*}, l_{1}, \lambda_{2}^{*}\left(l_{1} ; x_{1}^{*}\right)\right) d l_{1}
$$

exists, is continuous in $x_{1}^{*}$, and is increasing in $x_{1}^{*}$. The monotonicity is strict by the assumption of the strict state monotonicity A1.

Similarly, if we denote $\tilde{F}_{2}^{-1}(l, \eta)$ the inverse function to $\tilde{F}(z, z+\eta)$ with respect to $z$, we obtain $\vartheta_{2}\left(l_{2}\right)=x_{1}^{*}-\sigma \tilde{F}_{2}^{-1}\left(l_{2}, \eta\right)$ and $\lambda_{1}\left(l_{2}\right)=\tilde{F}\left(\tilde{F}_{2}^{-1}\left(l_{2}, \eta^{*}(\sigma)\right),-h_{2}\right)$. Again, both are continuous in $x_{1}^{*}$ and $\sigma$ and in the limit $\sigma \rightarrow 0$ we obtain $\vartheta_{2}\left(l_{2}\right) \rightarrow x_{1}^{*}$ and $\lambda_{1}\left(l_{2}\right)=\tilde{F}\left(\tilde{F}_{2}^{-1}\left(l_{2}, \eta^{* *}\right),-h_{2}\right)$ for $x_{1}^{*}$ considered in the case (c). Therefore, also the limit $D_{2}^{*}\left(x_{1}^{*}\right)=\lim _{\sigma \rightarrow 0} D_{2}\left(x_{1}^{*} ; \sigma\right)$ exists, and it is continuous in $x_{1}^{*}$. Moreover $D_{2}^{*}\left(x_{1}^{*}\right)$ is nondecreasing because we established in the Proof of Lemma 3 that $D_{2}\left(x_{1}^{*} ; \sigma\right)$ increases in 
$x_{1}^{*}$ for each $\sigma$. Therefore, the sum $m_{d y n}^{*}\left(x_{1}^{*}\right)=D_{1}^{*}\left(x_{1}^{*}\right)+D_{2}^{*}\left(x_{1}^{*}\right)$ exists, is continuous in $x_{1}^{*}$, and is increasing in $x_{1}^{*}$ for $x_{1}^{*}$ in the range considered in case $(\mathrm{c})$.

Summing up, we have established that the limit $m_{d y n}^{*}\left(x_{1}^{*}\right)$ is continuous in $x_{1}^{*}$ for ranges of $x_{1}^{*}$ considered in all the three cases (a), (b), and (c). Moreover, in case (c), $\eta^{* *}=-\left(h_{1}+h_{2}\right)$ if $u_{2}\left(x_{1}^{*}, 0,0\right)=0$, and $\eta^{* *}=h_{1}+h_{2}$ if $\int_{0}^{1} u_{2}\left(x_{1}^{*}, 1, l_{2}\right) d l_{2}=0$. This implies that $m_{d y n}^{*}\left(x_{1}^{*}\right)$ continuously connects at the boundaries in between the cases (a) and (c), and in between cases (c) and (b).

Lemma 7. (i) Action path 11 is selected at $\theta^{*}$ in $\Gamma_{\text {st }}$ if and only if $m_{s t}^{*}\left(\theta^{*}\right)>0$. Action 0 is selected at $\theta^{*}$ in $\Gamma_{\text {st }}$ if and only if $m_{s t}^{*}\left(\theta^{*}\right)<0$.

(ii) Action 11 or 10 is selected at $\theta^{*}$ in $\Gamma_{d y n}$ if and only if $m_{d y n}^{*}\left(\theta^{*}\right)>0$. Action 0 is selected at $\theta^{*}$ in $\Gamma_{d y n}$ if and only if $m_{d y n}^{*}\left(\theta^{*}\right)<0$.

Proof of Lemma 7. Claim (i) follows directly from Proposition 2.1 in Morris and Shin (2003) and from the assumption of bounded errors.

Claim (ii). By continuity of $m_{d y n}^{*}$, if $m_{d y n}^{*}\left(\theta^{*}\right)>0$ then $m_{d y n}^{*}(\theta)$ is positive on some $\delta$-neighborhood of $\theta^{*}$. Together with the monotonicity of $m_{d y n}(\theta ; \sigma)$ with respect to $\theta$ it implies that $m_{d y n}(\theta ; \sigma)$ is positive in a $\delta$-neighborhood of $\theta^{*}$ for $\sigma<\bar{\sigma}$, for some $\bar{\sigma}>0$. For sufficiently small $\bar{\sigma}$ all agents receive signals $x_{1}^{i}$ above $\theta^{*}-\delta$ in round 1 of $\Gamma_{d y n}(\sigma)$ whenever Nature draws fundamental $\theta^{*}$. Then, by Proposition 1 , all agents play action 1 in round 1 in the unique monotone symmetric equilibrium of $\Gamma_{d y n}(\sigma)$ (whenever Nature draws $\left.\theta^{*}\right)$.

A symmetric argument implies that if $m_{d y n}^{*}\left(\theta^{*}\right)<0$ then action 0 is selected.

Function $m_{d y n}^{*}(\theta)$ has a unique root at which none of the actions is selected in round 1 of $\Gamma_{d y n}$. Hence the reverse implications hold as well.

Let us now introduce and remind notation used in the proofs that follow; see Figure 9. Let $\bar{x}_{2}\left(x_{1}^{*} ; \sigma\right)=x_{1}^{*}+\sigma\left(h_{1}+h_{2}\right)$. If agent receives $\bar{x}_{2}\left(x_{1}^{*} ; \sigma\right)$ in round 2 , then she knows that all the fellow agents have received signals at least $x_{1}^{*}$ in round 1 . As before, $L_{2}\left(x_{1}^{*} ; \sigma\right)$ is the set of types who reach action path 11 if they play the rationalizable strategy in the continuation game $\Gamma_{2}\left(x_{1}^{*} ; \sigma\right)$, and $\mathbf{x}(\eta ; \sigma)$ is the intersection of $\partial L_{2}\left(x_{1}^{*} ; \sigma\right)$ with the line $x_{2}-x_{1}=\sigma \eta$, where $\eta \in\left[\underline{\eta}_{\Delta}, \bar{\eta}_{\Delta}\right]$. (The notation omits dependence of $\mathbf{x}(\eta ; \sigma)$ on $x_{1}^{*}$.) Let $x_{2}(\eta ; \sigma)$ be the second coordinate of $\mathbf{x}(\eta ; \sigma)$. We will pay attention to the round 2 signal $x_{2}\left(\bar{\eta}_{\Delta} ; \sigma\right)$ which has the following property implied by the monotonicity of $s_{2}$ : all types $\left(x_{1}, x_{2}\right) \in X$ such that $x_{1} \geq x_{1}^{*}$ and $x_{2} \geq x_{2}\left(\bar{\eta}_{\Delta} ; \sigma\right)$ reach action path 11 in $\Gamma_{2}\left(x_{1}^{*} ; \sigma\right)$.

Lemma 8. Assume that strategic uncertainty permits investment at $x_{1}^{*}$ in round 2, i.e., $\int_{0}^{1} u_{2}\left(x_{1}^{*}, 1, l_{2}\right) d l_{2}>0$. Then there exists $\bar{\sigma}>0$ such that $x_{2}\left(\bar{\eta}_{\Delta} ; \sigma\right)<\bar{x}_{2}\left(x_{1}^{*} ; \sigma\right)$ for all $\sigma<\bar{\sigma}$. 


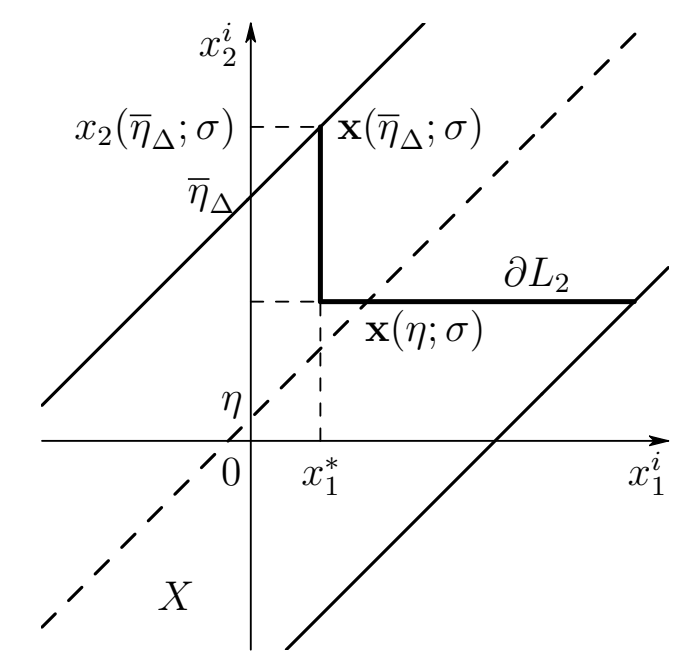

Figure 9: Additional notation.

Proof of Lemma 8. Suppose the contrary. Then there exists a sequence $\sigma_{k} \rightarrow 0$ such that $x_{2}\left(\bar{\eta}_{\Delta} ; \sigma_{k}\right) \geq \bar{x}_{2}\left(x_{1}^{*} ; \sigma_{k}\right)$ for all $k$. Let us explore beliefs of the type $\mathbf{x}\left(\bar{\eta}_{\Delta} ; \sigma_{k}\right)$. First, she knows that $\theta \geq x_{1}^{*}-\sigma_{k} h_{1}$. Second, she knows that $l_{1}=1$ because only fellow agents' signals at least $x_{1}^{*}$ are compatible with her second signal which is $\bar{x}_{2}\left(x_{1}^{*} ; \sigma_{k}\right)$ or larger. Third, her belief about $l_{2}$ stochastically dominates the uniform distribution on $[0,1]$. This is because all agents with $x_{2}^{i}>x_{2}\left(\bar{\eta}_{\Delta} ; \sigma_{k}\right)$ invest in both rounds and an agent receiving $x_{2}^{i}=x_{2}\left(\bar{\eta}_{\Delta} ; \sigma_{k}\right)$ has the uniform belief about the proportion of agents with the second signal above $x_{2}\left(\bar{\eta}_{\Delta} ; \sigma_{k}\right)$. Using all three observations we get

$$
U_{2}\left(\mathbf{x}\left(\bar{\eta}_{\Delta} ; \sigma_{k}\right)\right) \geq \int_{0}^{1} u_{2}\left(x_{1}^{*}-\sigma_{k} h_{1}, 1, l_{2}\right) d l_{2}
$$

The right-hand side of (18) is positive for sufficiently small $\sigma_{k}$ because $\int_{0}^{1} u_{2}\left(x_{1}^{*}, 1, l_{2}\right) d l_{2}$ is continuous in $x_{1}^{*}$. This contradicts the indifference condition $U_{2}\left(\mathbf{x}\left(\bar{\eta}_{\Delta} ; \sigma_{k}\right)\right)=0$.

Lemma 9. If strategic uncertainty permits investment at $\theta^{*}$ in round 2 and $m_{d y n}^{*}\left(\theta^{*}\right)>0$, then action path 11 is selected at $\theta^{*}$ in $\Gamma_{d y n}$.

Proof of Lemma 9. (See page 35 for notation.) As $m_{d y n}^{*}$ is continuous, $m_{d y n}^{*}\left(\theta^{*}-\delta\right)>0$ for some $\delta>0$. By Lemma 7 , action path 11 or 10 is selected at $\theta^{*}-\delta$. Thus, there exists $\bar{\sigma}>0$ such that for $\sigma<\bar{\sigma}$ all agents invest in round 1 of $\Gamma_{d y n}(\sigma)$ for all signals $x_{1}^{i} \geq \theta^{*}-\delta$. We can now apply Lemma 8: choosing $\delta>0$ small enough so that $\int_{0}^{1} u_{2}\left(\theta^{*}-\delta, 1, l_{2}\right) d l_{2}>0$, we have $s_{2}\left(\theta^{*}-\delta, \bar{x}_{2}\left(\theta^{*}-\delta ; \sigma\right)\right)=1$ in the unique equilibrium of $\Gamma_{d y n}(\sigma)$.

If Nature draws fundamental $\theta^{*}$ then types of all agents exceed $\left(\theta^{*}-\sigma h_{1}, \theta^{*}-\sigma h_{2}\right)$. We can choose $\bar{\sigma}^{\prime}$ small enough so that for $\sigma<\bar{\sigma}^{\prime},\left(\theta^{*}-\sigma h_{1}, \theta^{*}-\sigma h_{2}\right)>\left(\theta^{*}-\delta, \bar{x}_{2}\left(\theta^{*}-\delta ; \sigma\right)\right)$ and so that all agents invest in both rounds. 


\section{A.4 Proofs for Section 5}

Proof of Proposition 2. We showed that in the absence of backward spillovers: $m_{d y n}\left(x_{1}^{*} ; \sigma\right) \geq$ $m_{s t}\left(x_{1}^{*} ; \sigma\right)$ for any $x_{1}^{*}$ and $\sigma$, and so $m_{d y n}^{*}\left(x_{1}^{*}\right) \geq m_{s t}^{*}\left(x_{1}^{*}\right)$ for all $x_{1}^{*}$.

Claim (i). If action path 11 is selected at $\theta^{*}$ in $\Gamma_{s t}$ then, by Lemma $7,0<m_{s t}^{*}\left(\theta^{*}\right) \leq$ $m_{d y n}^{*}\left(\theta^{*}\right)$. By Lemma 9, action path 11 is selected at $\theta^{*}$ in $\Gamma_{d y n}$ because we assumed that strategic uncertainty permits investment at $\theta$ in round 2 .

Claim (ii). If 0 is selected at $\theta^{*}$ in $\Gamma_{d y n}$ then $0>m_{d y n}^{*}\left(\theta^{*}\right) \geq m_{s t}^{*}\left(\theta^{*}\right)$ and hence 0 is selected at $\theta^{*}$ in $\Gamma_{s t}$.

Proof of Proposition 3. We prove the two claims using the inequality

$$
m_{d y n}^{*}\left(x_{1}^{*}\right) \leq m_{s t}^{*}\left(x_{1}^{*}\right)
$$

which is established below.

Claim (i). If 11 is selected at $\theta$ in $\Gamma_{d y n}$ then $0<m_{d y n}^{*}(\theta) \leq m_{s t}^{*}(\theta)$ and so 11 is selected at $\theta$ in $\Gamma_{s t}$.

Claim (ii). If 0 is selected at $\theta$ in $\Gamma_{s t}$ then $0>m_{s t}^{*}(\theta) \geq m_{d y n}^{*}(\theta)$ and hence 0 is selected at $\theta$ in $\Gamma_{d y n}$.

We finish the proof by showing that if $\int_{0}^{1} u_{2}\left(x_{1}^{*}, 1, l_{2}\right) d l_{2}>0$ and payoffs do not exhibit forward spillovers then inequality (19) holds.

By Lemma 8, agents reach action path 11 in $\Gamma_{2}\left(x_{1}^{*} ; \sigma\right)$ whenever their round 2 signal exceeds $\bar{x}_{2}\left(x_{1}^{*} ; \sigma\right)$. Hence if Nature draws $\theta>\bar{x}_{2}\left(x_{1}^{*} ; \sigma\right)+\sigma h_{2}=x_{1}^{*}+\sigma\left(h_{1}+2 h_{2}\right)$ all agents reach action path 11 in $\Gamma_{2}\left(x_{1}^{*} ; \sigma\right)$.

Recall that we have defined $\vartheta_{t}\left(l_{t} ; x_{1}^{*}, \sigma\right)$ as the inverse function to the investment profile $l_{t}(\theta)$ induced by the rationalizable strategy in $\Gamma_{2}\left(x_{1}^{*} ; \sigma\right)$. Notice that $\vartheta_{t}\left(l_{t} ; x_{1}^{*}, \sigma\right)$ is non-decreasing in $l_{t}$, and, as follows from the previous paragraph, $\vartheta_{2}\left(l_{2} ; x_{1}^{*}, \sigma\right) \leq x_{1}^{*}+$ $\sigma\left(h_{1}+2 h_{2}\right)$. Using state monotonicity, A1, we have

$$
\begin{aligned}
\int_{0}^{1} u_{2}\left(\vartheta_{2}\left(l_{2} ; x_{1}^{*}, \sigma\right), l_{2}\right) d l_{2} & \leq \int_{0}^{1} u_{2}\left(x_{1}^{*}+\sigma\left(h_{1}+2 h_{2}\right), l_{2}\right) d l_{2} \leq \\
& \leq \int_{0}^{1} u_{2}\left(\vartheta_{1}\left(l_{2} ; x_{1}^{*}+2 \sigma\left(h_{1}+h_{2}\right), \sigma\right), l_{2}\right) d l_{2} .
\end{aligned}
$$

In the second inequality, we used $x_{1}^{*}-\sigma h_{1} \leq \vartheta_{1}\left(l ; x_{1}^{*}, \sigma\right)$ for all $l \in(0,1)$. This holds because $l_{1}(\theta)=0$ for $\theta \leq x_{1}^{*}-\sigma h_{1}$.

Furthermore, it follows from the inequality $l_{2}\left(l_{1}\right) \leq l_{1}$ and the action monotonicity 
A2 that

$$
\begin{aligned}
\int_{0}^{1} u_{1}\left(\vartheta_{1}\left(l_{1} ; x_{1}^{*}, \sigma\right), l_{1}, l_{2}\left(l_{1}\right)\right) d l_{1} & \leq \int_{0}^{1} u_{1}\left(\vartheta_{1}\left(l_{1} ; x_{1}^{*}, \sigma\right), l_{1}, l_{1}\right) d l_{1} \leq \\
& \leq \int_{0}^{1} u_{1}\left(\vartheta_{1}\left(l_{1} ; x_{1}^{*}+2 \sigma\left(h_{1}+h_{2}\right), \sigma\right), l_{1}, l_{1}\right) d l_{1}
\end{aligned}
$$

Summing the two inequalities we get $m_{d y n}\left(x_{1}^{*} ; \sigma\right) \leq m_{s t}\left(x_{1}^{*}+2 \sigma\left(h_{1}+h_{2}\right) ; \sigma\right)$ and, as $\sigma \rightarrow 0$, the inequality (19).

\section{A.5 Proof for Section 6}

Lemma 10. Consider two closed diagonally monotone sets $L \subseteq L^{\prime} \subseteq X$ and let $s, s^{\prime}$ : $X \longrightarrow\{0,1\}$ be their indicator functions. Assume that $\mathbf{s}=\left(s^{\prime}, s\right)$ satisfies properties $P 1$ and P2. Consider a function $U: X \rightarrow \mathbb{R}$ that is monotone in the sense of P1, continuous on the interior of $L^{\prime}$, and such that $L=\left\{\mathbf{x} \in L^{\prime}: U(\mathbf{x}) \geq 0\right\}$. Then

$$
E\left[s(\mathbf{x}) \cdot U(\mathbf{x}) \mid \partial L^{\prime}\right]=E[U(\mathbf{x}) \mid \partial L]
$$

Proof of Lemma 10. We decompose each of the expectations into two parts. Consider first the left-hand side of $(20)$ and recall that $s$ is the indicator function of the set $L:^{28}$

$$
\begin{aligned}
E\left[s(\mathbf{x}) \cdot U(\mathbf{x}) \mid \partial L^{\prime}\right]= & \operatorname{Pr}\left((X \backslash L) \mid \partial L^{\prime}\right) \cdot E\left[0 \cdot U(\mathbf{x}) \mid(X \backslash L) \cap \partial L^{\prime}\right] \\
& +\operatorname{Pr}\left(L \mid \partial L^{\prime}\right) \cdot E\left[1 \cdot U(\mathbf{x}) \mid L \cap \partial L^{\prime}\right] .
\end{aligned}
$$

Note that the first term is zero.

Consider now the right-hand side of (20):

$$
\begin{aligned}
E[U(\mathbf{x}) \mid \partial L]= & \operatorname{Pr}\left(\operatorname{int} L^{\prime} \mid \partial L\right) \cdot E\left[U(\mathbf{x}) \mid \operatorname{int} L^{\prime} \cap \partial L\right] \\
& +\operatorname{Pr}\left(L^{\prime \prime} \mid \partial L\right) \cdot E\left[U(\mathbf{x}) \mid L^{\prime \prime} \cap \partial L\right]
\end{aligned}
$$

where int $L^{\prime}=L^{\prime} \backslash \partial L^{\prime}$ and $L^{\prime \prime}=X \backslash\left(\operatorname{int} L^{\prime}\right)=\left(X \backslash L^{\prime}\right) \cup \partial L^{\prime}$. We show that the first term is again equal to zero and the second term is equal to the second term in (21). To see the former, recall that $L=\left\{\mathbf{x} \in L^{\prime}: U(\mathbf{x}) \geq 0\right\}$. Thus, by continuity, $U(\mathbf{x})=0$ for all $\mathbf{x} \in \partial L$ that lie in the interior of $L^{\prime}$. For the latter, observe that both $L^{\prime \prime} \cap \partial L$ and $L \cap \partial L^{\prime}$ are actually equal to $\partial L \cap \partial L^{\prime}$. Thus, the expectations in the second terms of (21) and (22) are identical.

\footnotetext{
${ }^{28}$ We define $\operatorname{Pr}\left(L \mid \partial L^{\prime}\right)=E\left[s(\mathbf{x}) \mid \partial L^{\prime}\right]$. Also expectation conditional on $L \cap \partial L^{\prime}$ is defined analogously as in the text preceding Lemma 1.
} 
It remains to show that $\operatorname{Pr}\left(L \mid \partial L^{\prime}\right)=\operatorname{Pr}\left(L^{\prime \prime} \mid \partial L\right)$. Recall that

$$
\begin{gathered}
\operatorname{Pr}\left(L \mid \partial L^{\prime}\right)=\lim _{d \rightarrow 0} \operatorname{Pr}\left(L \mid \partial L^{\prime d}\right)=\lim _{d \rightarrow 0} \operatorname{Pr}\left(L \cap \partial L^{\prime d}\right) / \operatorname{Pr}\left(\partial L^{\prime d}\right) . \\
\operatorname{Pr}\left(L^{\prime \prime} \mid \partial L\right)=\lim _{d \rightarrow 0} \operatorname{Pr}\left(L^{\prime \prime} \mid \partial L^{d}\right)=\lim _{d \rightarrow 0} \operatorname{Pr}\left(L^{\prime \prime} \cap \partial L^{d}\right) / \operatorname{Pr}\left(\partial L^{d}\right) .
\end{gathered}
$$

As argued in the proof of Lemma 1, the translational symmetry of the joint distribution of $\theta$ and $\mathbf{x}$ implies that for any diagonally monotone set $S$, diagonal translations of $\partial S^{d}$ occur with identical probabilities; that is, $\operatorname{Pr}\left(T_{d^{\prime}}\left(\partial S^{d}\right)\right)=\operatorname{Pr}\left(\partial S^{d}\right) .{ }^{29}$ This in turn implies that $\operatorname{Pr}\left(\partial S^{d}\right)=\frac{2 d}{\bar{\theta}-\theta}$ for boundary of any diagonally monotone set.

Therefore, $\operatorname{Pr}\left(\partial L^{\prime d}\right)=\operatorname{Pr}\left(\partial L^{d}\right)$ and $\operatorname{Pr}\left(L \cap \partial L^{\prime d}\right)=\operatorname{Pr}\left(L^{\prime \prime} \cap \partial L^{d}\right)$, where the second inequality holds as in fact $L \cap \partial L^{\prime d}=T_{d}\left(L^{\prime \prime} \cap \partial L^{d}\right)$. This completes the proof.

\section{References}

[1] Abreu, D., and Brunnermeier M.K., 2003. Bubbles and Crashes, Econometrica 71, $173-204$.

[2] Angeletos, G.M., Hellwig, C., and Pavan, A., 2006. Signaling in a Global Game: Coordination and Policy Traps, Journal of Political Economy 114, 452-484.

[3] Angeletos, G.M., Hellwig, C., and Pavan, A., 2007. Dynamic Global Games of Regime Change: Learning, Multiplicity and Timing of Attacks, Econometrica 75, $711-756$.

[4] Angeletos, G.M., and Werning, I., 2006. Crises and Prices: Information Aggregation, Multiplicity, and Volatility, American Economic Review 96, 1720-1736.

[5] Brunnermeier, M.K., and Morgan J., 2010. Clock Games: Theory and Experiments, Games and Economic Behavior 68, 532-550.

[6] Burdzy, K., Frankel D., and Pauzner, A., 2001. Fast Equilibrium Selection by Rational Players Living in a Changing World, Econometrica 68, 163-190.

[7] Carlsson, H., and van Damme, E., 1993. Global Games and Equilibrium Selection, Econometrica 61, 989-1018.

[8] Chamley, C., and Gale, D., 1994. Information Revelation and Strategic Delay in a Model of Investment, Econometrica 62, 1065-1085.

\footnotetext{
${ }^{29}$ The equation holds for such $d$ and $d^{\prime}$ that the relevant sets do not intersect with the boundary regions of the state space.
} 
[9] Chassang, S., 2010. Fear of Miscoordination and the Sustainability of Cooperation in Dynamic Global Games with Exit, Econometrica 78, 973-1006.

[10] Corsetti, G., Dasgupta, A., Morris, S., and Shin, H.S., 2004. Does One Soros Make a Difference: a Theory of Currency Crises with Large and Small Traders, Review of Economic Studies 71, 87-114.

[11] Dasgupta, A., 2007. Coordination and Delay in Global Games, Journal of Economic Theory 134, 195-225.

[12] Dasgupta, A., Steiner, J., and Stewart, C., 2012. Dynamic Coordination with Individual Learning, Games and Economic Behavior 74, 83-101.

[13] Dixit, A., and Pindyck, R., 1994. Investment under Uncertainty. Princeton: Princeton U. Press.

[14] Edmond, C., 2008. Information Manipulation, Coordination and Regime Change, mimeo, NYU Stern School of Business.

[15] Frankel, D.M., Morris, S., and Pauzner, A., 2003. Equilibrium Selection in Global Games with Strategic Complementarities, Journal of Economic Theory 108, 1-44.

[16] Gale, D., 1995. Dynamic Coordination Games, Economic Theory 5, 1-18.

[17] Goldstein, I., Ozdenoren, E., and Yuan, K., 2011. Learning and Complementarities in Speculative Attacks, Review of Economic Studies 78, 263-292.

[18] Goldstein, I., and Pauzner, A., 2005. Demand Deposit Contracts and the Probability of Bank Runs, Journal of Finance 60, 1293-1327.

[19] Gul, F., and Lundholm, R., 1995. Endogenous Timing and the Clustering of Agents' Decisions, Journal of Political Economy 103, 1039-1066.

[20] Heidhues, P., and Melissas, N., 2006. Equilibria in a Dynamic Global Game: the Role of Cohort Effects, Economic Theory 28, 531-557.

[21] Hörner, J., 2004. Payoff-Dominance in Dynamic Coordination Games, Mimeo.

[22] Kováč, E. and Steiner, J., 2008, Reversibility in Dynamic Coordination Problems, University of Edinburgh, discussion paper no. 183.

[23] Levin, J., 2001. A Note on Global Equilibrium Selection in Overlapping Generations Games, Mimeo, Stanford University.

[24] Mathevet, L., and Steiner, J., 2011. Sand in the Wheels: A Dynamic Global Game Approach, Mimeo, Norhtwestern University. 
[25] McDonald R., and Siegel D., 1986. The Value of Waiting to Invest, Quarterly Journal of Economics 101, 707-727.

[26] Milgrom, P., and Roberts, J., 1990. Rationalizability, Learning, and Equilibrium in Games with Strategic Complementarities, Econometrica 58, 1255-1277.

[27] Morris, S., and Shin, H.S., 1998. Unique Equilibrium in a Model of Self-Fulfilling Currency Attacks. American Economic Review 88, 587-597.

[28] Morris, S., and Shin, H.S., 2003. Global Games: Theory and Applications. In: Dewatripont, M., Hansen, M., Turnovsky, S. (Eds), Advances in Economics and Econometrics (Proceedings of the Eighth World Congress of the Econometric Society), Cambridge University Press.

[29] Morris, S., and Shin, H.S., 2004. Coordination Risk and the Price of Debt, European Economic Review 48, 133-153.

[30] Sákovics J., and Steiner J., 2012. Who Matters in Coordination Problems, American Economic Review, forthcomming.

[31] van Zandt, T., and Vives, X., 2007. Monotone Equilibria in Bayesian Games of Strategic Complementarities, Journal of Economic Theory 127, 339-360. 